\title{
Static Hedges for Reverse Barrier Options with Robustness Against Skew Risk: An Empirical Analysis
}

\author{
Jan H. Maruhn* Morten Nalholm ${ }^{\dagger} \quad$ Matthias R. Fengler ${ }^{\ddagger}$
}

\begin{abstract}
We conduct an empirical evaluation of a static super-replicating hedge of barrier options. The hedge is robust to uncertainty about the future skew. Using almost seven years of current data on the DAX, we evaluate the performance of the hedge and compare it with those of both a dynamic and a static replicating hedge. The main result is that the robustness of the static super-replicating portfolio is also empirically confirmed in practice such that the hedge sets an upper bound for the price of skew risk for barrier options.
\end{abstract}

\section{Introduction}

Reverse barrier options are barrier options that are in-the-money when they knock in or out. This means that reverse barrier options have a discontinuous payoff profile. If a knock-out event occurs the maximum intrinsic value is lost. As a result, dynamic hedging of reverse barrier options requires both frequent rebalancing and taking very large positions when the underlying is close to the barrier. This problem becomes particularly critical as time to expiry decreases. From a financial engineering and sales perspective, however, the discontinuous payoff profile allows for the construction of very attractive retail products. Hedging of reverse barrier options therefore is of considerable practical importance.

The literature on hedging barrier options by replication of the barrier payoff has two main branches, dynamic hedging and (semi-)static hedging. Dynamic hedging aims at continuously forming a riskless portfolio. In models where the market is assumed to be complete, such as the

\footnotetext{
*UniCredit Markets \& Investment Banking, HypoVereinsbank, Financial Engineering Equities, Commodities and Funds. jan.maruhn@unicreditgroup.de

$\dagger$ Department of Mathematical Sciences, University of Copenhagen nalholm@math.ku.dk

${ }^{\ddagger}$ Sal. Oppenheim jr. \& Cie., Untermainanlage 1, 60329 Frankfurt. This article reflects the authors’ personal opinion and not necessarily the opinion of their employers.

The authors gratefully acknowledge the support from and helpful discussions with Bernd Engelmann, Alexander Giese and Peter Schwendner as well as fruitful comments of two anonymous referees.
} 
Black \& Scholes (1973) model (BS), and the local volatility model (LV) introduced by Dupire (1994) and Derman \& Kani (1994), the riskless portfolio is formed by a position in the underlying and in a money market account. In practice, the assumptions of these models are violated, a fact that motivates ad-hoc hedging of other risk-factors like e.g. the volatility parameter in the BS model. The empirical performance of dynamic hedging of reverse barrier options using the LV model and hedging higher order sensitivities is investigated in Engelmann, Fengler \& Schwendner (2008).

Static hedging aims at replicating the barrier option payoff by taking positions in a number of vanilla options when the hedge is initiated. The hedge portfolio is unwound in the case of a knock-out event and otherwise held to expiry. Under certain assumptions this strategy achieves payoff replication. Two related methods have appeared in the literature starting with Derman, Ergener \& Kani (1995) and Carr \& Chou (1997). In those papers, static hedges are constructed for the BS model. In Nalholm \& Poulsen (2006b) these methods and their extensions to more general asset dynamics are unified and analyzed.

Comparisons of the performance of static and dynamic hedges mainly consist of simulation studies such as Tompkins (2002) and Nalholm and Poulsen (2006a,b). A recent empirical comparison is made in Engelmann, Fengler, Nalholm \& Schwendner (2006). One main conclusion is that some static hedges perform better than their dynamic counterparts, but that both types of strategies experience significant hedge errors as well as significant dispersion in these errors when applied in realistic setups.

To prevent potential losses, a natural idea is to consider hedging strategies which super-replicate the barrier option payoff. Regarding static hedging, the super-replication approaches in the literature can be divided into model-independent and model-dependent strategies. Model-independent hedges like the one proposed by Brown, Hobson \& Rogers (2001) guarantee super-replication of the barrier option payoff in any market environment. Unfortunately these hedges tend to be prohibitively expensive. In contrast, model-dependent static super-replicating hedges are usually much cheaper (see e.g. Giese \& Maruhn (2007)), but carry the risk of potential losses due to deviations of model and market prices. However, the risk of such deviations is significant, because the static hedge positions are computed at initiation of the hedge and afterwards kept constant until the barrier is hit or the option expires. It is very likely that the market environment at liquidation time differs substantially from the one at the time the hedge is set up, which in turn may result in hedging losses. Consequently, model-dependent static hedges for reverse barrier options carry significant model risk as for example illustrated by Hirsa, Courtadon \& Madan (2002) and Nalholm \& Poulsen (2006a).

To circumvent this problem, Maruhn \& Sachs (2008) recently developed a robustified static hedge in a stochastic volatility model, which reduces model dependence by making the hedge robust to significant changes in the model parameters. Practically speaking this means that the hedge guarantees the super-replication property for an infinite number of potential future volatility surface scenarios including changes of the at-the-money term structure, the smile and the skew. To a large extent, this key feature removes the significant (re)calibration risk experienced in markets; see Detlefsen \& Härdle (2007) and Cont (2006) for an investigation of this issue. The 
resulting robust hedge portfolios are much cheaper than the model-independent static hedges. They can therefore be thought of as a robust compromise between model-dependent and modelindependent static hedging strategies. However, while this concept is theoretically appealing it has remained unclear how this particular hedging strategy performs under real market conditions.

In this paper we close this gap by analyzing the empirical performance of the robust static superhedge on a seven years data set. To be more precise we monitor the hedge error for upand-out calls and down-and-out puts written on the DAX index and initiated at each day of the considered data set. Since the market prices of the hedge instruments change each day within the sample, one would expect that it is necessary to recompute the portfolio weights for static hedges of barrier options issued on different days. However, we are able to show that the robust static super-replication method allows to derive minimal assumptions hedges which are not only robust against changes of the volatility surface, but also independent of the hedge instrument prices at initiation. This enables us to set up the same static hedge (in the sense of absolute portfolio weights) for all barrier options issued throughout the sample.

Our empirical analysis shows that the minimal assumptions hedge is truly robust against changes of the volatility surface. Under the assumption that the hedge portfolio is liquidated on the barrier, the super-replication property holds in any market environment covered by the seven years data set. For the case of up-and-out calls the hedge is super-replicating even in the presence of moves beyond the barrier in the sample. For down-and-out puts the larger magnitude of downward moves leads to rare, but extreme hedge errors. This finding shows that hedges of reverse barrier options have to be robustified against skew and gap risk. As a benchmark, we compare our results to the strike spread approach of Carr \& Chou (1997) and a delta-vega hedge based on the local volatility model. As we find, the minimal assumptions hedge provides the smallest dispersion of the hedge error at an acceptable additional cost.

The paper is structured as follows. In Section 2 we describe the data set of volatility surfaces on the DAX used for the empirical analysis. As part of the description, we emphasize the main properties that are problematic when encountered by replication strategies. Section 3 describes the robust static super-replicating hedge developed in Maruhn \& Sachs (2008). The replicating strategies we use for comparisons are described in Section 4. We report the results in Section 5 , while conclusions and an outline of extensions are collected in Section 6.

\section{Market Data}

The data set consists of daily implied volatility data for vanilla options on the DAX traded at the EUREX. The sample period is January 2000 to November 2006 1] There are 1,752 observations in the sample. The options have European exercise and belong to the most liquid index contracts traded in Europe. Since the DAX is a capital weighted performance index, see Deutsche Börse

\footnotetext{
${ }^{1}$ The observed implied volatility corresponds to the last traded price an a given day. To prevent stale prices the exchange establishes an official settlement price if the last traded price is older than 15 minutes or if for some reason it is deemed not to reflect prevailing market conditions.
} 
(2006), dividends can be set to zero when valuing options on the DAX.

To clean the data, implied volatilities of options with less than 10 days to expiry are removed from the sample as are implied volatilities exceeding 80\%. Our dynamic hedging benchmark will make use of an LV model and it is therefore vital that there are no arbitrage opportunities in the cleaned data set. To prevent this, the data are smoothed in an arbitrage-free way using the procedure in Fengler (2008). For interest rates, the daily zero coupon rates are linearly interpolated as is commonly done, see e.g. Dumas, Fleming \& Whaley (1998).

The level of the DAX and the 1-year at-the-money (ATM) implied volatilities are shown in Figure 1. The sample exhibits periods with a trending market, periods with high as well as low volatility and periods with extreme movements like the 8.89\% drop on September 11, 2001. The level of both the short and long ( 1 year) end of the yield curve varies between $2 \%$ and $5 \%$ in the sample period.

\section{[Figure [1 about here]}

When considering reverse barrier options, it is not only the implied volatility level that is of interest. An up-and-out call option (UOC) can be decomposed into a vanilla call capped at the barrier and a short position in a one-touch option struck at the barrier, see Gatheral (2006) for an exposition. The latter is sensitive to the ATM skew of the implied volatility surface if the spot is close to the barrier. In Figure 2, we plot the term structures of the average, minimum, maximum and $2.5 \%$ as well as $97.5 \%$ quantiles of the ATM skew of the implied volatility surface. Figure 2 also shows the time series of daily observations for the 1-week and 1-year ATM skews in the implied volatility.

\section{[Figure 2 about here]}

The stable flattish skew term structure at the long end as well as the volatile steeply sloped short end of the skew term structure are consistent with the stylized facts of option markets on equity indices, see e.g. Chap. 2.7 in Fengler (2005). From the graph it can be seen that models, which do not capture this high variation in the short term skew, fail to mimic a very real phenomenon, even if they can match the average term structure. Popular equity models such as the Heston (1993) stochastic volatility (HSV) model

$$
\begin{aligned}
d S_{t} & =r(t) S_{t} d t+\sqrt{V_{t}} S_{t} d W_{t}^{1}, \\
d V_{t} & =\kappa\left(\theta-V_{t}\right) d t+\sigma \sqrt{V_{t}} d W_{t}^{2}, \\
d W_{t}^{1} d W_{t}^{2} & =\rho d t \\
d M_{t} & =r(t) M_{t} d t
\end{aligned}
$$

and the jump-diffusion stochastic volatility models e.g. by Bates (1996), Bakshi, Cao \& Chen (1997), Eraker, Johannes \& Polson (2003) and Eraker (2004) are too rigid to capture this variation 
in the skew, which is also typically found when fitting factor models to the implied volatility surface dynamics, such as Cont \& da Fonseca (2002) and Fengler, Härdle \& Mammen (2007). This is particularly problematic for hedges of reverse barrier options, which are highly exposed to the skew term structure. In response to this issue, a stochastic skew model has been introduced in Carr \& Wu (2007) for foreign-exchange markets.

The shortcomings of typical models to capture features like the variation in the implied volatility skew has led to the near-universal practice of recalibration. Calibration of models such as the HSV model is an inverse problem and as such requires some regularization, see Gerlich, Giese, Maruhn \& Sachs (2006) or Bouchouev \& Isakov (1999) for a review of the LV case. In Figure 3 we plot the parameter time series for the HSV model calibrated to the daily DAX implied volatility surfaces. Vega-weighted implied volatilities are used in the cost-function. Within the calibration, we enforce the Feller condition $\kappa \theta-\sigma^{2} / 2 \geq 0$, which ensures that $V_{t}>0$ for all $t$ with probability one. Furthermore we follow the suggestion of Bergomi (2004) in fixing $\kappa=2$, which serves as a regularization technique for the calibration problem. In 1622 out of 1750 cases the calibrated parameters satisfy the Feller condition with equality, which is a typical finding for highly skewed equity index markets.

\section{[Figure 3 about here]}

Our parameter tracks are in accordance with the results in Bergomi (2004) who calibrates to the EuroStoxx 50 index. It is worthwhile noting the qualitative agreement between the relative changes of the one week ATM volatility skew in Figure 2 and those in the time series for $\rho$ in Figure 3. This is because $\rho$ in the HSV model captures the skew2. Similarly, there is a correspondence between the one year ATM volatility in Figure 1 and $V_{0}$ in Figure 3. Again, this is to be expected since $V_{0}$, together with $\theta$, captures the volatility level. Overall, the instable parameter tracks simply reflect the fluctuations of the market.

There is an obvious inconsistency in the recalibration practice. If the calibrated parameters change this is equivalent to adopting a new model at each recalibration. From Figure 3, it is clear that hedges based on a recalibrated HSV model are likely to face substantial deterioration of the hedge performance even if one tries to hedge model parameter changes in the sense of sensitivities.

Making a hedge strategy robust to such parameter changes is of major importance to avoid the hedge errors caused by the changing market conditions. A possible solution would be the uncertain volatility approach due to Avellanda, Levy \& Parás (1995). Here, the BS model is made robust to uncertainty about the volatility parameter. However, since static hedge portfolios consist of options with several strikes and expiries, one cannot ignore the overall shape of the implied volatility surface and only focus on the (current and future) instantaneous volatility as does the uncertain volatility approach. Maruhn \& Sachs (2008) therefore suggested a robust

\footnotetext{
${ }^{2}$ The volatility of variance $\sigma$ also has an impact on the skew, since setting $\sigma=0$ leads to a flat surface in strike direction. This can be inferred from the smile expansions in terms of the Heston parameters as derived in Gatheral (2006), pp. 35. Given its stronger variation, see Figure 3, $\rho$ is the dominating skew parameter.
} 
static super-replication technique, which takes variations of skew and smile parameters explicitly into account. This approach will be described and empirically analyzed in the next sections.

\section{Robust Super-Replicating Hedges}

In this section we describe and analyze the robust static super-replication approach which forms the basis for our empirical analysis. After introducing the concept in Subsection 3.1, we examine the cost of a successive robustification in Subsection 3.2 before discussing the hedge stability with respect to changing market prices at initiation of the hedge in Subsection 3.3 .

\subsection{Introduction of Concept}

For convenience we introduce the static super-replication method developed in Maruhn \& Sachs (2008) for the case of a reverse up-and-out call (UOC) without rebates and constant barrier. However, reverse down-and-out puts (DOP), rebates and non-constant barriers can be treated in analogy (see Maruhn (2009) for details). The description uses the HSV model, see Equation (1), but the method works for general stochastic volatility dynamics including Hull \& White (1987), Stein \& Stein (1991) and Hagan, Kumar, Lesniewski \& Woodward (2002).

To be more precise we are interested in hedging an UOC with expiry $T>0$, strike $K>0$, barrier $B>S_{0}$ and payoff

$$
U O C(T):=\left(S_{T}-K\right)^{+} 1_{\left\{\max _{t \in[0, T]} S_{t}<B\right\}} .
$$

Since the UOC is reverse we have that the barrier exceeds the strike, i.e. $B>K$. We denote the value of the barrier option at time $t$ by $U O C(t)$.

In order to hedge the UOC we set up a portfolio of $N$ call options $C^{i}, i=1, \ldots, N$, with strikes $K^{i}$, expiries $T^{i} \leq T$ and payoff $C^{i}\left(T^{i}\right):=\left(S_{T^{i}}-K^{i}\right)^{+}$. In contrast to dynamic replication strategies the portfolio weights $\alpha^{i}$ of the calls are not continuously adjusted during the lifetime of the barrier option. Instead they are kept constant until either the barrier $B$ is breached or the UOC expires. Regarding these two cases it is a natural idea to include the call with the same strike and expiry as the UOC in the hedge portfolio, because it guarantees a perfect hedge in case of no barrier hit, and to choose additional calls with strikes $K^{i} \geq B$ and expiries $T^{i} \leq T$ which reduce the value of the portfolio to account for the discontinuity in the payoff function at the barrier.

In the HSV model (1) the value $C^{i}(t)$ of call $C^{i}$ at time $t$ depends on the current spot $S_{t}$ and variance $V_{t}$ such that $C^{i}(t)=C^{i}\left(t, S_{t}, V_{t}\right)$. Furthermore, calls with expiries $T^{i}<T$ (which are assumed to satisfy $K^{i} \geq B$ ) expire worthless if the barrier has not been hit up to time $T^{i}$. Correspondingly, in case of no barrier breach before time $t$, the value of the portfolio of calls $C^{i}$, 
$i=1, \ldots, N$, at time $t$ is given by

$$
\Pi(\alpha ; t)=\Pi\left(\alpha ; t, S_{t}, V_{t}\right):=\sum_{i \in\{1, \ldots, N\}: T^{i} \geq t} \alpha^{i} C^{i}\left(t, S_{t}, V_{t}\right) .
$$

This static hedge portfolio is super-replicating if it provides a payoff greater than or equal to the UOC in the two cases of a barrier hit or no barrier hit until expiry $T$. Observing that the payoff (2) of the UOC is either equal to zero or $\left(S_{T}-K\right)^{+}$, we can represent the super-replication property in the HSV model by the set of constraints

$$
\begin{gathered}
\sum_{i \in\{1, \ldots, N\}: T^{i} \geq t} \alpha^{i} C^{i}\left(t, S_{t}, V_{t}\right) \geq 0 \quad \forall\left(t, S_{t}, V_{t}\right) \text { for which the barrier might be hit } \\
\sum_{i \in\{1, \ldots, N\}: T^{i}=T} \alpha^{i}\left(S_{T}-K^{i}\right)^{+} \geq\left(S_{T}-K\right)^{+} \quad \forall S_{T} \text { that can be attained without a barrier hit. }
\end{gathered}
$$

Clearly the stock price $S_{t}$ in the HSV model can hit the barrier $B$ at any time $t \in(0, T]$. Furthermore, if the correlation $\rho$ between the Brownian motions driving $S_{t}$ and $V_{t}$ satisfies $|\rho|<1$, the variance $V_{t}$ can attain any value in $(0, \infty)$ at that time. Finally note that the stock price $S_{T}$ can reach any value in $(0, B)$ without previously hitting the barrier. Hence the super-replication property in the HSV model can be rephrased as

$$
\begin{gathered}
\sum_{i \in\{1, \ldots, N\}: T^{i} \geq t} \alpha^{i} C^{i}(t, B, v) \geq 0 \quad \forall(t, v) \in(0, T] \times(0, \infty) \\
\sum_{i \in\{1, \ldots, N\}: T^{i}=T} \alpha^{i}\left(s-K^{i}\right)^{+} \geq(s-K)^{+} \quad \forall s \in(0, B) .
\end{gathered}
$$

The optimal superhedge in the HSV model is characterized as the portfolio $\alpha$ with minimal cost $\Pi(\alpha ; 0)$ satisfying the constraints (3). This hedge can be computed at time $t=0$ in a two step process. First we calibrate the parameters of the HSV model (1) to the implied volatility surface at time $t=0$. In the following we denote the resulting parameters by $V_{0}, \kappa_{0}, \theta_{0}, \sigma_{0}$ and $\rho_{0}$. Afterwards we solve the linear semi-infinit 3 optimization problem

$$
\min _{\alpha \in \mathbb{R}^{n}} \Pi(\alpha ; 0)=\sum_{i=1}^{N} \alpha^{i} C^{i}\left(0, S_{0}, V_{0}\right) \quad \text { s.t. Constraints (3) }
$$

by applying a suitable numerical optimization algorithm. However, while the solution of problem (4) guarantees the super-replication property within the HSV model, the hedge performance may suffer significantly in a real world setting. This can best be seen by observing that the constraints (3) only guarantee the super-replication property if the calibrated Heston parameters at the time of a barrier hit equal $v, \kappa_{0}, \theta_{0}, \sigma_{0}$ and $\rho_{0}$ with arbitrary start variance $v \in(0, \infty)$. But this means that the portfolio is only protected against movements of the short term volatility $\sqrt{v}$, whereas the other parameters characterizing the volatility surface are assumed to remain constant over time. Figure 3 clearly demonstrates that this assumption is violated in practice. The shape of the volatility surface changes over time and so do the implied HSV parameters, which

\footnotetext{
${ }^{3}$ The optimization problem is called semi-infinite since the minimization of $\Pi(\alpha, 0)$ is carried out with respect to an infinite number of constraints.
} 
in turn might lead to significant hedging losses for purely model-dependent hedge portfolios like the one solving problem (41).

Maruhn \& Sachs (2008) quantified the risk due to neglected changes of the model parameters for the static hedge portfolio solving (4) and showed that potential hedge errors can amount to multiples of the fair value of the barrier option (which similarly applies to all other modeldependent static hedge approaches in the literature). Based on the insights above the authors proposed the natural solution to further robustify the static hedge by asking the super-replication property (3) to also hold for (simultaneous) changes of all parameters of the HSV model. This leads to the robust optimization problem

$$
\begin{gathered}
\min _{\alpha \in \mathbb{R}^{n}} \Pi(\alpha ; 0)=\sum_{i=1}^{N} \alpha^{i} C^{i}\left(t, S_{0}, V_{0}, \kappa_{0}, \theta_{0}, \sigma_{0}, \rho_{0}\right) \\
\text { s.t. } \sum_{i \in\{1, \ldots, N\}: T^{i} \geq t} \alpha^{i} C^{i}(t, B, v, \kappa, \theta, \sigma, \rho) \geq 0 \quad \forall t \in[0, T] \forall p:=(v, \kappa, \theta, \sigma, \rho) \in P, \\
\sum_{i \in\{1, \ldots, N\}: T^{i}=T} \alpha^{i}\left(s-K^{i}\right)^{+} \geq(s-K)^{+} \quad \forall s \in[0, B]
\end{gathered}
$$

where the dependence of the call option prices on all model parameters is explicitly expressed through the notation $C^{i}(0)=C^{i}\left(0, S_{0}, V_{0}, \kappa_{0}, \theta_{0}, \sigma_{0}, \rho_{0}\right)$ and $C^{i}(t)=C^{i}(t, B, v, \kappa, \theta, \sigma, \rho)$, respectively. Further $P \subset \mathbb{R}^{5}$ denotes a suitable set of model parameters corresponding to markettypical volatility surface scenarios.

Figure 3 reveals that each of the model parameters seems to vary within a typical range which motivates to choose $P$ as a multi-dimensional interval $\left[v_{\min }, v_{\max }\right] \times\left[\kappa_{\min }, \kappa_{\max }\right] \times\left[\theta_{\min }, \theta_{\max }\right] \times$ $\left[\sigma_{\min }, \sigma_{\max }\right] \times\left[\rho_{\min }, \rho_{\max }\right]$. However, to exclude parameter combinations for which the variance process in the HSV model reaches zero with positive probability, we intersect this interval with the set of points satisfying the Feller condition $\kappa \theta-\sigma^{2} / 2 \geq 0$. This final set $P$ represents the future volatility surface scenarios in which the static hedge solving problem (5) is guaranteed to super-replicate the UOC. The choice of $P$ therefore directly addresses recalibration risk and eliminates it within the specified parameter intervals.

Although this approach breaks with the constant model parameter assumption of the HSV model, it is possible to prove the existence of an optimal static hedging strategy solving (5) under the mild assumption that the feasible set is non-empty, see Maruhn \& Sachs (2008). In applications this is trivially fulfilled, because the call with the same strike and expiry as the barrier option is included as a hedge instrument. Moreover, an optimal strategy can be computed by solving a sequence of linear and nonlinear optimization problems. In the following we briefly sketch the idea of the numerical algorithm. For details including a convergence proof we refer to Maruhn \& Sachs (2008).

In order to reduce the infinite number of constraints in problem (5) to a numerically tractable quantity, we first replace the sets $[0, T] \times P$ and $[0, B]$, namely the volatility surface scenarios $p \in P$ at the time of the barrier hit $t \in[0, T]$ as well as the stock price scenarios $s \in[0, B]$ in case of no barrier hit, with discrete approximations $M_{1}, M_{2}$ consisting of a finite number of points. Afterwards we compute the hedge portfolio $\alpha$ which super-replicates the barrier option in these scenarios $(t, p) \in M_{1}, s \in M_{2}$ by solving the linear optimization problem (5) with the 
finite number $\left|M_{1}\right|+\left|M_{2}\right|$ constraints. Neglecting the remaining scenarios $([0, T] \times P) \backslash M_{1}$ and $[0, B] \backslash M_{2}$ leaves room for potential hedge errors in case the realized scenario differs from those contained in $M_{1}, M_{2}$. To verify this we compute the worst case hedge error for the portfolio $\alpha$ by minimizing the constraints in (5) over the whole sets $[0, T] \times P$ and $[0, B], \alpha$ fixed, with a standard nonlinear optimization algorithm. Afterwards we add the scenario(s) for which this worst case hedge error is realized to the sets of scenarios $M_{1}, M_{2}$ and recompute the hedge which guarantees super-replication with respect to this extended set of scenarios. This linear/nonlinear programming cycle is repeated iteratively until convergence is achieved. Usually this is the case in less than ten iterations or five minutes of computing time on a $3 \mathrm{GHz}$ desktop PC.

Although the additional robustness of the hedge is appealing, the reduction of recalibration risk will not come for free. In the next section we analyze how cost and composition of the optimal portfolio solving problem (5) change if we successively robustify the hedge against changes of the at-the-money term structure, the smile and the skew.

\subsection{Step-Wise Robustification}

The definition (5) of the static superhedge raises the question how the weights $\alpha^{i}$ and cost $\Pi(\alpha ; 0)$ change if we robustify the portfolio against different features of the implied volatility surface. In the following this will be investigated by performing the robustification in three steps as indicated in Table 1. The first step (A) basically robustifies against changes in the level of the implied volatility surface in both the short and long term. The hedge is made robust to the three parameters $\sqrt{v}, \kappa, \sqrt{\theta}$ in one step since the portfolio is quite stable at each incremental step. The second step (B) additionally robustifies against changes in the smile of the implied volatility surface. Finally, the third step (C) adds robustness against changes in the implied volatility skew.

\section{[Table 1 about here]}

The initial parameter values $p_{0}=\left(\sqrt{V_{0}}, \kappa_{0}, \sqrt{\theta_{0}}, \sigma_{0}, \rho_{0}\right)=(25 \%, 200 \%, 26 \%, 50 \%,-70 \%)$ and the parameter intervals are chosen to reflect typical values for the DAX. This can be seen from the tracks of calibrated parameter values in Figure 3. Note that the intervals do not encompass all observations of the parameters since this would be equivalent to an unrealistic foresight in our empirical application. As described in Subsection 3.1 the set $P$ is chosen as the intersection of the parameter hypercube listed in Table 1 with the set of points satisfying the Feller condition.

The robust hedges are constructed for an UOC with expiry $T=1$ year, strike at $90 \%$ and barrier at $120 \%$ of the level of the underlying asset price, and a down-and-out put (DOP) with the same expiry, strike at $110 \%$ and barrier at $80 \%$. Furthermore, we choose the initial spot $S_{0}=100 \%$ and a constant riskfree interest rate of $r=3 \%$. To hedge the UOC, we make use of the ten standard calls listed in Table 2 which were liquidly traded on the EUREX in January 2006. In analogy we set up a portfolio of ten puts with appropriate strikes/expiries to hedge 
the DOP. The strikes for both the UOC and DOP hedges were identified by first computing the robust static super-replicating hedge for a large set of traded plain vanilla options, which was then successively reduced to the relevant instruments with non-zero portfolio weights. The strike difference of $1.8 \%$ roughly corresponds to an absolute difference of 100 index points in January 2006.

Table 2 reports the optimal portfolios and the cost corresponding to the stepwise robustifications A - C. It can be seen that the hedge portfolios essentially consist of three components. Naturally, a position in the vanilla with the same strike and expiry as the barrier option is taken. This hedges perfectly in the absence of a knock-out event. Secondly, a large negative payoff with the same expiry as the barrier option is constructed just beyond the barrier. The negative time value of this payoff balances the positive payoff from the underlying vanilla along the barrier. Thirdly, small positions are taken in the instruments with expiry before the barrier option. They trim the time values of the payoffs expiring at the same time as the barrier option. The same components were also used in previous papers on static hedge portfolios without robustness against changes of the volatility surface, see e.g. Nalholm \& Poulsen (2006b) or Giese \& Maruhn (2007).

\section{[Table 2 about here]}

Table 2 reveals that the composition of the hedges changes considerably across the steps. In particular it can be seen that the positions forming the call/put spread become smaller when increasing the robustness against changes of the volatility surface. The more protection we want to incorporate in the hedge, the less aggressive we have to choose the positions. In addition, the portfolio weights of the options with expiry before the expiry date of the barrier option drop as well. Among the three proposed robustification steps A, B, and C, the protection against changes in correlation $\rho$ in step $\mathrm{C}$, i.e. against changes in the skew, appear to have the most significant impact for the UOC, whereas the DOP is more affected by the volatility of variance $\sigma$.

This differing behavior can be understood by analyzing the impact of the Heston parameters on the dominating digital positions at the barrier, which is approximated by call and put spreads. For the UOC the hedge portfolio contains a large negative position in the call $C^{2}$ with strike $K^{2}=120.0$ and a large positive position in the call $C^{3}$ with higher strike $K^{3}=121.8$. The value of this call spread diminishes if the cost of call $C^{2}$ increases relative to the cost of call $C^{3}$. This occurs when the slope of the implied volatility surface becomes more negative in strike direction (in trader's language: the digital position is short the skew). Hence the critical volatility surface scenarios for the static hedge of the UOC are those with steep skew, which in turn corresponds to very negative values of the correlation parameter $\rho$. Thus a robustification against the parameter $\rho$ can be expected to have the most significant effect on the portfolio positions and on the costs of the hedge portfolio. This intuition is confirmed by the results in Table 2. For the DOP the situation is reversed. In this case the large negative position is taken in the put with strike 80.0, which is offset by a huge positive position in the put with lower strike 78.8. Thus the critical volatility surface scenarios for the DOP are those with an almost flat downside of the volatility 
surface in strike direction 4 . Within the HSV model, these scenarios are reflected by a small volatility of variance parameter $\sigma$. Hence robustifying against changes of $\sigma$ is more important than against variations of $\rho$. Table 2 illustrates these effects in form of the changes of the cost and portfolio positions throughout the robustification steps $\mathrm{A}, \mathrm{B}$ and $\mathrm{C}$.

In summary, the robust static hedge guarantees the super-replication property for the critical states of the forward skew. This is a conservative hedging approach as can be confirmed by comparing the cost of the static super-replication portfolios listed in Table 2 with the prices of the barrier options in the HSV and the LV model. For the HSV model with parameters $p_{0}$ the theoretical fair value of the UOC and DOP can be computed as $U O C_{H S V}(0)=4.22 \%$ and $D O P_{H S V}(0)=2.55 \%$. In contrast, a local volatility model calibrated to the volatility surface implied by the HSV model with parameters $p_{0}$ leads to the prices $U O C_{L V}(0)=3.36 \%$ and $D O P_{L V}(0)=2.79 \%$, respectively. The price differences illustrate the model risk for these instruments. Although both the HSV and the LV model are calibrated to the same volatility surface at time $t=0$, the differing model dynamics lead to very different assumptions regarding the forward skew. It is well known, see Gatheral (2006), that the forward skew is flattening quickly in LV models, whereas it is approximately time-homogeneous in stochastic volatility models. But this means that the digital risk of the DOP is priced more conservatively in a $\mathrm{LV}$ model $\left(D O P_{L V}(0)=2.79 \%>2.55 \%=D O P_{H S V}(0)\right)$, whereas the steeper forward skew of the HSV model produces higher prices for the up-and-out call $\left(U O C_{L V}(0)=3.36 \%<4.22 \%=\right.$ $\left.U O C_{H S V}(0)\right)$. In comparison to these dynamic hedging approaches the robust static hedge does not make a particular assumption regarding the forward skew. Instead it robustifies the portfolio against all sorts of future volatility surfaces reflected by the parameters in $P$. This more conservative viewpoint comes at a fairly low cost. Comparing with Table 2 , it can be seen that the UOC static hedge is only 50 basis points more expensive than the HSV option price (4.22\% versus $4.72 \%$ for the UOC static hedge). Similarly, the DOP robust static hedge only requires an additional 30 basis points on top of the cost of the more conservative LV option price $(2.79 \%$ versus $3.09 \%$ for the DOP static hedge).

The arguments above illustrate that the robust static hedge is up to a certain degree modelindependent. In particular the model generating the volatility surface scenarios (in our case the HSV model) does not matter. If two models parameterize the same set of volatility surfaces, the resulting static hedge will be the same. However, the Heston model is particularly convenient for the construction of the hedge since it only has a few parameters steering the shape of the implied volatility surface. The results presented in this subsection showed that in general the inclusion of all five model parameters in the robustification is necessary. Therefore, the remainder of the paper focusses on analyzing the super-replicating hedges corresponding to robustfication step C in Table 2,

\footnotetext{
${ }^{4}$ Equity markets are usually not faced with a positive skew. Within the HSV model, this would lead to positive correlations $\rho$ which we excluded from the parameter hypercube described in Table 1.
} 


\subsection{Sensitivity Analysis With Respect to Initial Cost}

The robustification approach has the advantage of preserving the super-replicating property of a hedge under changing market conditions. However, the algorithm finds the cost-optimal portfolio with respect to initial market prices when the hedge is constructed. These market prices can be expressed by the calibrated parameters $p_{0}=\left(\sqrt{V_{0}}, \kappa_{0}, \sqrt{\theta_{0}}, \sigma_{0}, \rho_{0}\right)$ of the HSV model at time $t=0$. But this means that different market prices (or parameters $p_{0}$ ) in general lead to different super-replication hedges for barrier options with identical specifications set up on different days, even when the hedges are robustified against the same parameter hypercube. Thus, some of the appeal of the robust hedge is lost since the dependence on the concurrent calibration remains.

The observations above raise questions about the sensitivity of the robust static hedge against changes of the initial parameter vector $p_{0}$. In the following we analyze this question for the hedge of the UOC listed in step $\mathrm{C}$ in Table 2. To be more precise, we fix the parameter hypercube $P$ corresponding to step $\mathrm{C}$ in Table 1 and recompute the optimal hedge for the UOC using random initial parameter vectors, $p_{i}, i=1, \ldots, 200$, drawn uniformly and independently from the parameter hypercube $P$ (in an elementwise sense). Clearly all these hedge portfolios possess the super-replicating property as long as the market can be represented by a vector from $P$. Moreover, if current market prices are reflected by the initial parameter $p_{i}$, the costs of the optimal hedge corresponding to $p_{0}$, but evaluated at $p_{i}$, must be greater or equal to the costs of the optimal hedge computed for $p_{i}$. What remains to judge is the magnitude of the sub-optimality cost of the $p_{0}$-hedge in this situation.

In Figure 4, the cost of the sub-optimality in basis points, Cost $_{0}-$ Cost $_{i}$, based on market prices obtained from $p_{i}$, is shown. The left panel shows the cost as a function of the overall distance between the fixed $p_{0}$ and the random $p_{i}$. The right panel shows the cost as a function of the largest relative parameter value deviation from the value in $p_{0}$. From the graphs it can be seen that although $P$ represents quite diverse market conditions the cost of sub-optimality is (often much) smaller than 25 basis points. The mean cost of sub-optimality is a mere 4 basis points. It is not clear that a larger overall difference between two market scenarios, $\left|p_{i}-p_{0}\right|_{2}$, leads to larger costs. Rather the relative deviation of a single element in $p_{i}$ appears to determine the cost of sub-optimality.

\section{[Figure 4 about here]}

The previous observations allow to conclude that, using a fixed parameter vector despite changing market conditions, one only incurs small additional costs. The average sub-optimality of 4 basis points shows that the initial market prices have a small impact on the robust static hedge if compared to that of choosing the parameter hypercube $P$. This allows us to neglect changes of the market prices and instead choose a fixed parameter $p_{0}$ for the computation of the hedge throughout the considered data set. The implications are far-reaching. Fixing the parameter $p_{0}$ in problem (5) implies that we have a hedge portfolio where the portfolio weights are fixed even though the market changes. This is possible because the optimization problem (5) is homogeneous in the asset price level at the start date of the barrier option when all contract specifications of 
the target option and hedge instruments, such as strikes and barriers, are chosen relative to this level. The hedge portfolio retains the super-replicating property as long as the market can be described by a vector in $P$, that is $P$ is the only assumption we make about the parameters. We therefore refer to the portfolios listed in step $\mathrm{C}$ in Table 2 as minimal assumptions (MA) hedge portfolios. The MA hedges will allow us in Section 5 to set up static hedge portfolios with the same absolute portfolio weights for the barrier options issued on each day of six years of data.

\section{Replicating Hedges and Experimental Design}

An empirical evaluation of the performance of the robust super-replicating hedge is valuable in its own right. However, a comparison with replication strategies typically found in the literature gives the empirical investigation considerably more relevance. To evaluate the performance of a hedge strategy one should focus on the hedge errors and particularly their dispersion. In the following we describe the replicating hedges and the way in which the time series of realized hedge errors are constructed.

\subsection{Replicating Hedges}

To judge the performance of the robust hedge we compare it with two replicating hedge strategies, a standard dynamic hedge as well as a replicating static hedge. For the dynamic hedge a large variety of models are available. In this paper we choose a dynamic hedge based on the localvolatility model, because this kind of models is frequently used in practice and differs from the HSV model we used to set up the static hedge.

The hedge performance of the LV model for the reverse barrier problem is analyzed in Engelmann et al. (2008) who focus on the 'stickiness assumptions' on the implied volatility dynamics. When computing the sensitivity of the option price with respect to movements in the underlying three methods are frequently used in practice. First, one can assume that the local volatility surface remains fixed. This is consistent with the LV model, but not with the observed market behavior. Second, one can suppose that the implied volatility surface remains fixed. This is called the sticky-strike approach. Finally, one can presume that the implied volatility surface floats with the price of the underlying. This is called the sticky-moneyness approach. Stickiness assumptions are ad-hoc ways to capture the dynamics of the implied volatility surface. One main result of Engelmann et al. (2008) is that the sticky-strike assumption is superior to the others, but differences are small when a vega hedge is added to the hedge portfolio.

The dynamic hedge we consider is a sticky-strike delta-vega hedge (DV) in the LV model, that is we hedge both against shifts in the underlying and (parallel) shifts of the implied volatility surface. To compute the vega the implied volatility surface is shifted and the sensitivity is found by recalibration and a finite difference approximation. Engelmann et al. (2008) find the DV hedge to be considerably better than a pure delta hedge and only slightly less effective than a 
delta-vega-vanna hedge. To hedge the delta and vega, positions are taken in the money market account, the spot and the ATM vanilla option with the same expiry as the barrier option. The hedge strategy is rebalanced daily.

The second alternative hedging strategy is the strike-spread approach (STR) developed in Carr \& Chou (1997) based on the BS model and refined in Carr, Ellis \& Gupta (1998). The idea is to match the value of the payoff from the barrier option, by the negative value of a reflected version of the payoff at maturity, such that the value of the total payoff is zero along the barrier. This adjusted payoff function is then approximated to arbitrary precision by taking positions in vanilla options. We do not consider pure calendar spreads by Derman et al. (1995) because they are known to be of inferior quality, see Engelmann et al. (2006). Carr et al. (1998) show that in the BS model

$$
d S_{t}=(r-q) S_{t} d t+\sigma_{B S} S_{t} d W_{t},
$$

the adjusted payoff functions for the UOC and DOP, are given as

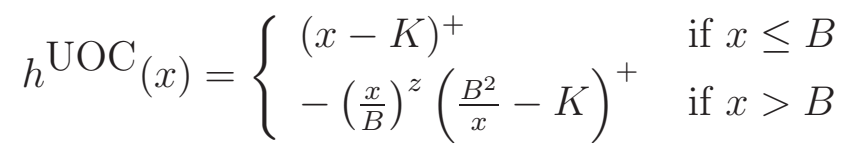

and

$$
h^{\mathrm{DOP}}(x)= \begin{cases}(K-x)^{+} & \text {if } x>B \\ -\left(\frac{x}{B}\right)^{z}\left(K-\frac{B^{2}}{x}\right)^{+} & \text {if } x \leq B\end{cases}
$$

where $z=1-2(r-q) / \sigma_{B S}^{2}$. Examples of the payoff functions $h$ are sketched in Figure 5 for the case $r-q=0$.

\section{[Figure [5 about here]}

Constructing an expiry- $T$ portfolio of strike-spreads that approximates $h$, at the initiation of the barrier option and holding it to either a barrier breach or expiry constitutes a replicating static hedge. Although the STR hedge is static like the MA hedge, it is neither super-replicating nor robust with respect to changes of the model parameters. Finally, note that the STR hedge is constructed under the BS model which does not have an implied volatility skew.

To get an idea of the expected performance of the STR hedge in the presence of an implied volatility skew, one can for example consider an anologous static hedge in the Bachelier model

$$
d S_{t}=\sigma_{\text {Bachelier }} d W_{t}
$$

with zero risk neutral drift. Even though this model produces a negative implied volatility skew, the Gaussian asset price distribution 5 allows to derive an exact replicating static hedge given by the adjusted payoff functions

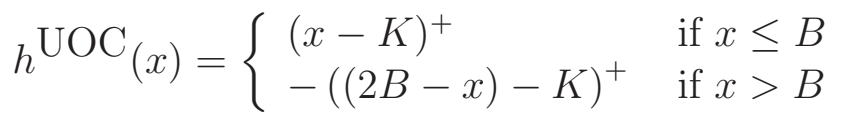

\footnotetext{
${ }^{5}$ The issues of negative asset prices in the Bachelier model are not relevant in our setting since we are only interested in the volatility surface scenarios generated by the model if the stock price is at the barrier.
} 
and

$$
h^{\mathrm{DOP}}(x)= \begin{cases}(K-x)^{+} & \text {if } x>B \\ -(K-(2 B-x))^{+} & \text {if } x \leq B\end{cases}
$$

Figure 5 shows that there is a larger interval with a negative payoff under the BS than under the Bachelier model in the UOC case and a smaller interval with a negative payoff under the BS model in the DOP case. Since a negative implied volatility skew is present in the data, as seen from Figure 2, the BS-based STR hedge will hence tend to produce positive hedge errors in the DOP case and negative hedge errors in the UOC case.

\subsection{Experimental Design}

For each day we initiate short positions in a reverse up-and-out call and a reverse down-and-out put. For each contract we set up long positions in the robust MA hedge, a static STR hedge as well as the dynamic DV hedge. The hedges are monitored daily with the dynamic hedge being rebalanced using a recalibrated LV model. If the rebalancing of the dynamic hedge is not self-financing the money market account is adjusted to reflect this. The money market account is rolled at the short-term interest rate of the relevant day.

A hedge error, that is the difference between the payoff from the hedge and that of the corresponding barrier option at knock-out or expiry, is recorded. This is done for each of the first 1,500 days in the sample which means that all barrier options may run to expiry in the absence of a knock-out event. This design results in time series of realized hedge errors using market data for each of the three hedges and each barrier option type (UOC/DOP). To make the hedge errors comparable, all levels referring to the DAX are in percent of the DAX at initiation of the particular barrier option.

As in Section 3, the UOC has strike at $90 \%$ and a barrier at $120 \%$ of the DAX. For the DOP the numbers are $110 \%$ and $80 \%$ respectively. Correspondingly, the STR hedge uses as hedge options calls with strikes at $\left\{90 \%, 120 \%, 121.8 \%,\left(B^{2} / K\right)=160 \%\right\}$ in the UOC case, whereas puts with strikes $\left\{110 \%, 80 \%, 78.8 \%,\left(B^{2} / K\right)=58.2 \%\right\}$ are used for the DOP hedge. The hedge instruments and portfolio weights used for the robust super-replicating hedges are those listed for step $\mathrm{C}$ in Table 2. The strike difference of $1.8 \%$ roughly corresponds to the ratio of 50 index points (the strike spacing of DAX index options with expiry less than one year on the EUREX) to the minimum of the DAX path in the sample. Hence $1.8 \%$ is a larger spread difference than would effectively have been attainable for most of the time during the sample period.

When closing a hedge, the hedge error is rolled at short-term interest rates until expiry. If no knock-out event occurs for a given barrier option, the hedge is unwound at observed market prices and the hedge error is recorded. In case the barrier is hit, a choice must be made regarding the spot at which the hedge is closed. One possibility is to unwind the hedge at the observed spot beyond the barrier. This is consistent with the data, but inconsistent with the hedge strategies since these require the hedge to be closed exactly when the knock-out event occurs. Therefore, we also consider closing the hedges exactly at the barrier but assuming that the observed implied 
volatilities apply at this time. Effectively, the latter approach shifts the spot from the observed level to the barrier. This is (often slightly) inconsistent with the data, but consistent with the hedge strategies. A point in favor of choosing the first approach is that it is unlikely that a hedge can be unwound exactly at the knock-out event in practice. Using the spot beyond the barrier can be seen as using a proxy for this gap risk. Closing the hedges on the barrier however focuses on the skew sensitivity of the barrier options. Hence, both approaches are relevant and we report results for both.

\section{Results}

To be able to properly interpret the time series of hedge errors, information on the knock-out events must be considered. The number of knock-out events as well as the average life time of both types of barrier options are reported in Table [3. From the table it can be seen that the DOP case will be harder to hedge. More DOP than UOC contracts knock-out after a shorter average life time. Furthermore, the moves beyond the barrier are larger on average in the DOP case.

\section{[Table 3 about here]}

The first question to consider is whether the super-replicating property of the robust hedges holds empirically if we assume that the hedge portfolios are closed on the barrier. To answer this the realized hedge errors for both the UOC and DOP are shown in Figure 6. It is seen that the super-replicating property is confirmed empirically. This implies that the market conditions prevailing at the knock-out dates correspond or are close to some parameter vector in $P$, at least for the hedge options.

\section{[Figure 6 about here]}

Next, the frequencies of hedge errors corresponding to the MA, STR and dynamic hedges of the UOC are compared in Figure 7 (left). From the graph it can be seen that a main advantage of static hedges with an STR-like component is that a perfect hedge is obtained in the absence of a knock-out event. This is not the case for dynamic hedges where the hedge portfolio must be closed at market prices at expiry. This difference explains the large peak of the hedge error distribution at zero shared by the MA and STR hedges. The super-replication property of the robust MA hedge is found as before. For the STR hedge the interval where the hedge has a large negative intrinsic value is considerably wider than for the MA hedge. This, as well as the lack of positive payoff beyond the barrier and the implied volatility skew, leads to negative hedge errors in almost all knock-out events. This is as expected. The hedge errors for the MA and STR hedges are almost mirror images of each other reflected through zero. The hedge errors of the DV hedge on the other hand are uni-modal with a fat positive tail. It is noteworthy that 
the dynamic hedge generates a larger number of positive hedge errors than the MA hedge, even though the MA hedge was constructed without direct penalty for large infrequent hedge errors.

\section{[Figure 7 about here]}

Similarly, the frequencies of hedge errors for the three hedge strategies in the DOP case are shown, see Figure 7 (right). Again, the peak of the hedge error distribution at zero is shared by both the MA and STR hedges corresponding to a perfect hedge when there is no knock-out event. Apart from the peak at zero, the positive hedge errors from the MA hedge are closer to zero than those from the STR hedge. This shows the strength of the approximate cost-optimality of the super-replicating MA hedge. The nonzero hedge errors from the STR hedge are positive as expected when taking implied volatility skew and average undershoot into account.

Summary statistic: 6 for the different hedges are given in Table 4. The sensitivity of the results for the STR hedge emphasizes the fragility of this particular hedging strategy. The lack of consistent performance of STR-like hedges was one of the observations in Engelmann et al. (2006). Comparing the STR outcome with the MA results gives an indication of the performance of the new static hedging strategy. For the UOC the MA hedge only leads to 3 observations of slightly negative hedge errors, which are all smaller than $4 \mathrm{bp}$ in absolute value. This underlines the robustness of the MA hedge against future changes of the implied volatility surface including the skew.

\section{[Table 4 about here]}

In the DOP case the mean cost (computed from market prices) for this robustness is surprisingly low, in particular if the potential worst case loss of $-6.35 \%$ of the DV hedge is taken into account. In the UOC case the cost of the MA hedge on first sight seems to be significantly higher than of the other hedges. However, recalling our analysis from Subsection 3.2, the higher cost is caused by differing assumptions regarding the dynamics of the volatility surface. The quickly flattening forward skew of the LV model prices the digital risk contained in the UOC less conservatively than the MA hedge. Table 4 reveals that the more conservative assumptions of the MA hedge also lead to much more conservative UOC hedge error statistics compared to the other hedging strategies. In this context we mention that the cost of the MA hedge would in fact be closer to the cost of a (more conservative) dynamic hedge in a stochastic volatility model. However, in the DOP case the DV hedging cost and the price of the MA hedge are much closer to each other, because in this situation a flat forward skew is the more conservative scenario.

In the rest of this section we focus on the errors generated by unwinding at the observed spot after the barrier breach, that is, using the data consistent approach. The reason for this is that

\footnotetext{
${ }^{6}$ Note that the numbers for the STR and DV hedges in the table deviate somewhat from those reported in Engelmann et al. (2006). There are two reasons for this. First, the sample has been lengthened and more knockouts occur. This affects both the DV and the STR hedges. Second, the strike of hedge option $i=3$ in the STR has been changed to $K^{3}=121.8 \%$. This is done for the MA and STR hedges to share this hedge option.
} 
we believe gap risk could well be an issue when attempting to unwind potentially large vanilla positions. Furthermore, this approach does not favor the robust MA hedge unjustly relative to the replicating hedges.

Figure 8 illustrates that the super-replication property still holds for the UOC in this case. However, for the DOP the data consistent hedge closing approach leads to 38 negative hedge errors 6 of which are below $-4 \%$, which is approximately the size of the mean hedge cost. The three very large hedge errors occur when the knock-out event happens close to expiry and the spot moves to about $78 \%$ of spot at initiation, that is just beyond the barrier. In these cases, the large short position in the $80 \%$ put option dominates the payoff. Relative to the sample size of 1,500 contracts only a small number generates negative hedge errors. A few of them, however, are spectacular.

\section{[Figure 8 about here]}

This observation is confirmed by the summary statistics presented in Table 5. For the UOC the super-replication property of the MA hedge is found as before. There are only 3 negative hedge errors leading to losses which are all smaller than $30 \mathrm{bp}$ in absolute value. In case of the DOP the MA hedge still has mainly positive hedge errors, but the potentially extreme losses with low probability are clearly visible. These large losses are also shared by the STR hedge, which is expected since both hedging approaches construct a steep negative payoff area at maturity to offset the intrinsic value of the barrier option. In contrast the DV hedge avoids the spectacular negative errors.

\section{[Table 5 about here]}

Remarkably, the dispersion of the MA hedge is still very low in the presence of both skew and gap risk. The measure of dispersion we consider is mean absolute deviation about the median ('mad.'), which is a more robust measure than the standard deviation ('std.') to estimate the variability of non-symmetric distributions. In fact the static MA hedge has the lowest dispersion for both barrier option types, particularly in the UOC case. This result is surprising since we actually used the same portfolio weights for each MA hedge set up within the 1500 day sample.

In summary, we can conclude for both the UOC and DOP that the robust static portfolio succeeds in hedging the skew risk, but that the DOP hedge is still exposed to gap risk. This is not a surprise taking the bigger gap risk for the DOP into consideration, see Table 3 , and recalling that the optimization problem (5) assumes the liquidation of the hedge portfolio on the barrier by construction. Although it is in principle possible to additionally robustify the MA hedge against gap risk (see Maruhn (2009)), we did not pursue this approach here, because we did not apply similar techniques for the DV and STR hedge. In reality, it is reasonable to expect the MA hedges to be closed somewhere in between the barrier and the closing price at the day of the barrier breach. This will tend to make negative hedge errors less frequent. Finally, note that static hedges with an STR-like component, such as the STR and MA hedges are relatively 
insensitive to large negative moves, such as the $8.89 \%$ drop on 11 September 2001. If the hedge is closed at the closing price, then the intrinsic value of the hedge is close to zero, since the spot has leaped the strike-interval where the hedge has a large negative payoff.

Another aspect to consider are the bounds sub- and super-replicating hedges put on fair barrier option prices. Since we have near-perfect super-replication the cost of the MA hedge provides an upper bound on the fair prices of UOC and DOP contracts, respectively. A lower bound can be found by solving the corresponding sub-replication problem

$$
\begin{gathered}
\max _{\alpha \in \mathbb{R}^{n}} \Pi(\alpha ; 0)=\sum_{i=1}^{N} \alpha^{i} C^{i}\left(t, S_{0}, V_{0}, \kappa_{0}, \theta_{0}, \sigma_{0}, \rho_{0}\right) \\
\text { s.t. } \sum_{i \in\{1, \ldots, N\}: T^{i} \geq t} \alpha^{i} C^{i}(t, B, v, \kappa, \theta, \sigma, \rho) \leq 0 \quad \forall t \in[0, T] \forall p:=(v, \kappa, \theta, \sigma, \rho) \in P, \\
\sum_{i \in\{1, \ldots, N\}: T^{i}=T} \alpha^{i}\left(s-K^{i}\right)^{+} \leq(s-K)^{+} \quad \forall s \in[0, B] .
\end{gathered}
$$

We solve (6) using the initial parameter values and robustness intervals specified in step C in Table 1, The hedge instruments and corresponding optimal portfolio weights are listed in Table 6. Table 7 summarizes the empirical results. As can be seen from the maximum hedge errors the sub-replicating hedges achieve true sub-replication in the sample.

\section{[Table 6 about here]}

\section{[Table 7 about here]}

Combining the mean costs of the robust super- and sub-replicating MA hedges we obtain the cost bounds $[3.50 \%, 5.30 \%]$ for the UOC and $[1.38 \%, 3.38 \%]$ for the DOP (see Tables 5, 7). Note that the mean cost $3.94 \%$ of the DV hedge based on the LV model for the UOC is not too far away from the lower bound of the corresponding interval, which indicates that this particular strategy is an aggressive one for up-and-out calls. On the other hand, comparing the DOP cost of $2.69 \%$ of the DV hedge with the robust interval confirms that the hedge is much more conservative for down-and-out puts.

Finally, in order to understand how the alternative hedging strategies compare when taking risk aversion and cost of the hedge into account, we compute the expected utility for an agent with exponential utility, i.e. $U(X)=-\exp (-\lambda X)$, for various choices of $\lambda>0$. In our case $X$ denotes the difference of the hedge error minus cost of the hedge. For the exponential utility $\lambda$ has the interpretation of the Arrow-Pratt coefficient of constant absolute risk aversion. We use a utility function exhibiting constant absolute risk aversion, because we take the view of a trading desk. A trading desk typically has constant risk limits; thus, a utility with constant absolute risk aversion is more realistic than a utility with decreasing absolute risk aversion, which is usually preferred to model individuals, see Campbell (1999). In our computations we take the costs of the hedges into account and assume that the trader does not receive any premium for the short 
position in the option. Otherwise the risk-averse agent would always prefer the more conservative hedge which is trivial. The situation we envisage could be interpreted to the one of a back book trader who hedges the liability of the option without receiving any compensation. By varying the risk-aversion coefficient we obtain a preference relation from expected utility which allows for comparing the different trading strategies.

We first consider the rankings of the hedges when unwinding the hedge at the barrier (we do not display the tables for sake of brevity). For the UOC, for moderate values of risk aversion, i.e. $\lambda<2.5$, DV ranks before MA, while for bigger values of risk aversion MA ranks first with STR being always the least favourable strategy. For the DOP, for $\lambda<0.75$, STR ranks first, MA second and DV third. When $0.75<\lambda<6$, MA and STR permute ranks; for values of $\lambda>7$, i.e. extremely risk-averse agents, STR moves to the third place, while DV ranks second. Concluding, when the hedge is unwound at the barrier, the additional costs of the MA hedge are readily accepted in exchange for a better hedge when risk aversion is moderate.

For the case when the hedge is unwound at the observed spot results are less clear cut. For the DOP we find that the MA hedge ranks always third, while STR ranks first for values of $\lambda<0.75$; for larger values of $\lambda$ the DV ranks first. Clearly this is due to the spectacular hedging errors occurring for both static hedges, see Table 5. For the UOC, for values of $\lambda<6.5$, DV ranks before MA, while for $\lambda>6.5 \mathrm{MA}$ is ranked first with STR being always the least favourable strategy. It is thus first at very high levels of risk aversion only that the MA hedge is preferred (if at all). It is however remarkable that the DV hedge, which is a hedging strategy often pursued in practice, is not completely inferior under risk aversion when the distributions, which result from unwinding the hedge at the observed spot, and hedging costs are considered.

\section{Conclusion and Extensions}

In this paper we consider various approaches to hedging reverse barrier options. To the best of our knowledge we contribute to the literature with the first empirical investigation of the performance of a cost-optimal static super-replicating hedge using a large market data set. Furthermore, we provide a comparison with the performances of typical replicating hedges. Our results show that the robust static hedge succeeds in eliminating the skew and volatility risk of barrier options at an acceptable additional cost.

Apart from our empirical analysis we investigate the properties of the robust static hedge and its impact on recalibration risk from various perspectives. Our analysis shows that in contrast to typical dynamic hedging strategies the robust static super-replicating hedge neither takes a view on the forward skew nor on the volatility surface dynamics. Moreover, we show that the market price of the hedge instruments at initiation of the hedge only has a small impact on the cost of the portfolio. This shows that the robust hedge is mainly determined by the specifications of the barrier option and a conservative view on future volatility surface scenarios. The hedge automatically translates the market-typical variability of the implied volatility surface into a necessary degree of robustness that needs to be embedded in the hedge portfolio. The less 
stable the surface is over time, the more robustness we need and the more expensive the hedge is. However, compared to typical replicating hedges the costs are found to be low. Finally, by homogeneity of the options issued, the minimal assumptions hedge has the property that portfolio weights stay the same, even if the hedges correspond to barrier options issued on different days and in different market conditions.

Using seven years of daily data on the DAX index the performance of the minimal assumptions hedge, of a standard static hedge and of a dynamic replicating hedge are compared. The superreplicating property of the minimal assumptions robust hedge is found to hold for the whole data sample in the UOC case. For the DOP, the super-replicating property is limited to the case of liquidating the hedge portfolio on the barrier. If the DOP hedge is unwound at the observed spot, more negative hedge errors occur - a few of which are spectacular, but not greater than those incurred by replicating static hedging strategies. The results reflect the fact that hedging of reverse barrier options is a hard problem, which requires to take both skew and gap risk into account. The empirical investigation in this paper shows that the robust static hedge succeeds in eliminating the skew risk, and hence implies an upper bound for the price of this risk which is also relevant in the context of dynamic hedging. Combined with the associated sub-replicating minimal assumptions hedges, we find empirically robust price bounds for barrier options on the DAX index.

Our results motivate further empirical research on the performance of gap-robustified static and dynamic hedges. The theoretical foundation for a gap-robust version of the robust hedge is found in Maruhn (2009). The ad-hoc practice of 'moving the barrier' when hedging barrier options is the corresponding approach for replicating hedges. Clearly, hedge portfolios for down-and-out puts would benefit from such a robustification. Moreover, it would be interesting to compare the hedge performance of the minimal assumptions hedge and the delta-vega hedge in a local volatility model to a suitable dynamic hedge derived in a stochastic volatility model. Regarding the differing forward skew dynamics such a hedge can be expected to be closer to the MA hedge in the UOC case, whereas it should produce less conservative portfolios for the DOP. 


\section{References}

Avellanda, M., Levy, A. \& Parás, A. (1995), 'Pricing and Hedging Derivative Securities in Markets with Uncertain Volatilities', Aplied Mathematical Finance 2, 73-88.

Bakshi, G., Cao, C. \& Chen, Z. (1997), 'Empirical performance of alternative option pricing models', Journal of Finance 52(5), 2003-2049.

Bates, D. (1996), 'Jumps and stochastic volatility: Exchange rate processes implicit in deutsche mark options', The Review of Financial Studies 9(1), 69-107.

Bergomi, L. (2004), 'Smile dynamics', Risk Magazine pp. 117-123.

Black, F. \& Scholes, M. (1973), 'The pricing of options and corporate liabilities', Journal of Political Economy 81, 637-654.

Bouchouev, I. \& Isakov, V. (1999), 'Uniqueness, stability and numerical methods for the inverse problem that arises in financial markets', Inverse Problems 15, R95-R116.

Brown, H. M., Hobson, D. \& Rogers, L. C. G. (2001), 'Robust hedging of barrier options', Mathematical Finance 11, 285-314.

Campbell, J. Y. (1999), Asset prices, consumption, and the business cycle, in J. B. Taylor \& M. Woodford, eds, 'Handbook of Macroeconomics', Vol. 1, Elsevier, chapter 19, pp. 12311303.

Carr, P. \& Chou, A. (1997), 'Breaking Barriers', Risk Magazine 10, 139-145.

Carr, P., Ellis, K. \& Gupta, V. (1998), 'Static Hedging of Exotic Options', Journal of Finance 53, 1165-1190.

Carr, P. \& Wu, L. (2007), 'Stochastic skew for currency options', Journal of Financial Economics 86(1), 213-247.

Cont, R. (2006), 'Model uncertainty and its impact on the pricing of derivative instruments', Mathematical Finance 16(3), 519-547.

Cont, R. \& da Fonseca, J. (2002), 'Dynamics of implied volatility surfaces', Quantitative Finance $2,45-60$.

Derman, E., Ergener, D. \& Kani, I. (1995), 'Static Options Replication', Journal of Derivatives $2,78-95$.

Derman, E. \& Kani, I. (1994), 'Riding on a smile', Risk Magazine 7, 32-39.

Detlefsen, K. \& Härdle, W. K. (2007), 'Calibration risk for exotic options', Journal of Derivatives 14(4), 47-63.

Deutsche Börse (2006), Guide to the Equity Indices of Deutsche Börse, 5.12 edn, Deutsche Börse AG, 60485 Frankfurt am Main, Germany. 
Dumas, B., Fleming, J. \& Whaley, R. E. (1998), 'Implied Volatility Functions: Empirical Tests', Journal of Finance 53, 2059-2106.

Dupire, B. (1994), 'Pricing with a smile', Risk Magazine 7, 18-20.

Engelmann, B., Fengler, M. R., Nalholm, M. \& Schwendner, P. (2006), 'Static versus dynamic hedges: An empirical comparison for barrier options', Review of Derivatives Research 9(3), 239-264.

Engelmann, B., Fengler, M. R. \& Schwendner, P. (2008), 'Hedging under alternative stickiness assumptions: an empirical analysis for barrier options'.

Eraker, B. (2004), 'Do stock prices and volatility jump? reconciling evidence from spot and option prices', Journal of Finance 59(3), 1367-1403.

Eraker, B., Johannes, M. \& Polson, N. (2003), 'The impact of jumps in volatility and returns', Journal of Finance 63(3), 1269-1300.

Fengler, M. R. (2005), Semiparametric Modeling of Implied Volatility, Lecture Notes in Finance, Springer-Verlag, Berlin, Heidelberg.

Fengler, M. R. (2008), 'Arbitrage-free smoothing of the implied volatility surface', Quantitative Finance. Forthcoming.

Fengler, M. R., Härdle, W. \& Mammen, E. (2007), 'A semiparametric factor model for implied volatility surface dynamics', Journal of Financial Econometrics 5(2), 189-218.

Gatheral, J. (2006), The Volatility Surface, A Practitioner's Approach, John Wiley and Sons, Inc.

Gerlich, F., Giese, A., Maruhn, J. \& Sachs, E. (2006), Parameter identification in stochastic volatility models with time-dependent model parameters, Technical report, University of Trier.

Giese, A. M. \& Maruhn, J. H. (2007), 'Cost-optimal static super-replication of barrier options an optimization approach', Journal of Computational Finance 10(3), 71-97.

Hagan, P. S., Kumar, D., Lesniewski, A. S. \& Woodward, D. E. (2002), 'Managing Smile Risk', WILMOTT magazine 1(2), 84-108.

Heston, S. (1993), 'A Closed-Form Solution for Options with Stochastics Volatility with Applications to Bond and Currency Options', Review of Financial Studies 6, 327-344.

Hirsa, A., Courtadon, G. \& Madan, D. B. (2002), 'The Effect of Model Risk on the Valuation of Barrier Options', Journal of Risk Finance 4(Winter), 47-55.

Hull, J. \& White, A. (1987), 'The pricing of options on assets with stochastic volatilities', Journal of Finance 42(2), 281-300. 
Maruhn, J. H. (2009), Robust Static Super-Replication of Barrier Options, Vol. 7 of Radon Series on Computational and Applied Mathematics, de Gruyter.

Maruhn, J. H. \& Sachs, E. W. (2008), 'Robust static hedging of barrier options in stochastic volatility models', Mathematical Methods of Operations Research .

Nalholm, M. \& Poulsen, R. (2006a), 'Static Hedging and Model Risk for Barrier Options', Journal of Futures Markets 26(5), 449-463.

Nalholm, M. \& Poulsen, R. (2006b), 'Static hedging of barrier options under general asset dynamics: Unification and application', Journal of Derivatives 13(4), 46-60.

Stein, E. \& Stein, J. (1991), 'Stock price distributions with stochastic volatility: An analytic approach', Review of Financial Studies 4, 727-752.

Tompkins, R. G. (2002), 'Static versus Dynamic Hedging of Exotic Option: An Evaluation of Hedge Performance via Simulation', Journal of Risk Finance 3(Summer), 6-34. 


\begin{tabular}{|c|c|c|c|c|c|c|c|}
\hline & meter & $p_{0}$ & Lower bound & Upper bound & \multicolumn{3}{|c|}{ Step } \\
\hline 1 & $\sqrt{v}$ & 0.25 & 0.10 & 0.40 & \multirow{3}{*}{$\varangle$} & \multirow{4}{*}{$ص$} & \multirow{5}{*}{$\circlearrowright$} \\
\hline 2 & $\kappa$ & 2.00 & 1.50 & 2.50 & & & \\
\hline 3 & $\sqrt{\theta}$ & 0.26 & 0.20 & 0.30 & & & \\
\hline 4 & $\bar{\sigma}$ & 0.50 & 0.20 & 0.60 & & & \\
\hline 5 & $\rho$ & -0.70 & -0.90 & -0.40 & & & \\
\hline
\end{tabular}

Table 1: Heston parameter intervals used for the robustification. Step B comprises step A, step C comprises both A and B.

\begin{tabular}{|c|c|c|c|c|c|c|c|c|c|c|c|c|}
\hline \multirow{5}{*}{0} & $T^{i}$ & 1.0 & 1.0 & 1.0 & 1.0 & 1.0 & 0.75 & 0.75 & 0.75 & 0.5 & 0.5 & \\
\hline & $K^{i}$ & 90.0 & 120.0 & 121.8 & 125.4 & 130.8 & 120.0 & 123.6 & 130.8 & 120.0 & 127.2 & Costs \\
\hline & $\mathrm{A}$ & 1.0 & -36.09 & 41.70 & -7.72 & 1.30 & 0.31 & 0.24 & -0.76 & 0.06 & 0.13 & 4.33 \\
\hline & B & 1.0 & -34.22 & 37.51 & $\begin{array}{l}-3.72 \\
\end{array}$ & -0.61 & $\begin{array}{l}0.13 \\
\end{array}$ & 0.28 & -0.38 & 0.01 & 0.14 & 4.36 \\
\hline & $\mathrm{C}$ & 1.0 & -28.78 & 28.40 & 1.20 & -2.09 & 0.19 & -0.07 & 0.21 & 0.08 & -0.03 & 4.72 \\
\hline \multirow{5}{*}{ 命 } & 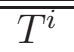 & $\overline{1.0}$ & $\overline{1.0}$ & $\overline{1.0}$ & $\overline{1.0}$ & $\overline{1.0}$ & 0.75 & 0.75 & 0.75 & 0.5 & $\overline{0.5}$ & \multirow[b]{2}{*}{ Costs } \\
\hline & $K^{i}$ & 110.0 & 80.0 & 78.8 & 76.4 & 72.8 & 80.0 & 77.6 & 72.8 & 80.0 & 75.2 & \\
\hline & $\mathrm{A}$ & 1.0 & -79.48 & 100.0 & -25.87 & 5.87 & -0.86 & 1.09 & -0.55 & 0.04 & -0.11 & 2.57 \\
\hline & B & 1.0 & -51.59 & 43.36 & 17.06 & -9.60 & 0.02 & 0.01 & 0.14 & -0.01 & 0.02 & 3.00 \\
\hline & $\mathrm{C}$ & 1.0 & -46.41 & 33.04 & 24.74 & -12.37 & 0.04 & -0.02 & 0.23 & -0.01 & 0.02 & 3.09 \\
\hline
\end{tabular}

Table 2: Hedge portfolio specifications in form of absolute portfolio weights $\alpha^{i}$ for different robustification steps. Hedge options are calls for UOC and puts for DOP. Strikes and costs (in the sense of HSV prices) are in percent of the level of the underlying.

\begin{tabular}{|c|c|c|c|}
\hline Option & Knock-out & Avg. life time & Avg. over-/undershoot \\
\hline UOC & $39.5 \%$ & 140 & $0.50 \%$ \\
DOP & $48.2 \%$ & 116 & $-1.78 \%$ \\
\hline
\end{tabular}

Table 3: Proportion of 1,500 barrier options that knocked out. The average life time is in days and the average over- and undershoots are relative to the barrier being crossed. 


\begin{tabular}{|l|l||r|r||r|r|r|r|r|r|r|r|}
\hline & Hedge & Mean cost & $P$ (Loss) & min & max & mean & med. & std. & mad. & skew. & kurt. \\
\hline \hline \multirow{2}{*}{$\circlearrowleft$} & MA & 5.30 & $0.20 \%$ & -0.04 & 30.0 & 1.0 & 0.0 & 1.7 & 1.0 & 4.6 & 64.8 \\
\cline { 2 - 12 } & STR & 3.48 & $37.33 \%$ & -4.67 & 30.0 & -0.9 & 0.0 & 1.6 & 1.0 & 3.9 & 86.1 \\
\cline { 2 - 12 } & DV & 3.94 & $59.53 \%$ & -3.87 & 26.2 & 0.1 & -0.3 & 2.7 & 1.5 & 4.0 & 30.1 \\
\hline \hline \multirow{2}{*}{$心$} & MA & 3.38 & $0.00 \%$ & 0.00 & 30.0 & 1.4 & 0.0 & 1.9 & 1.4 & 5.2 & 76.8 \\
\cline { 2 - 11 } & STR & 3.88 & $0.00 \%$ & 0.00 & 30.0 & 2.0 & 0.0 & 2.4 & 2.0 & 2.4 & 26.1 \\
\cline { 2 - 11 } & DV & 2.69 & $74.13 \%$ & -6.35 & 35.8 & -0.2 & -0.4 & 1.8 & 0.9 & 10.0 & 166.5 \\
\hline
\end{tabular}

Table 4: Summary statistics for all hedges in both the UOC and DOP cases if the hedge portfolio is unwound at the barrier $B$. The mean absolute deviation about the median is labelled 'mad.'. All numbers except skewness, kurtosis and the loss probability are in percent of spot at initiation. The costs are computed from market prices.

\begin{tabular}{|l|l||r|r||r|r|r|r|r|r|r|r|}
\hline & Hedge & Mean cost & $P($ Loss $)$ & min & max & mean & med. & std. & mad. & skew. & kurt. \\
\hline \hline \multirow{2}{*}{} & MA & 5.30 & $0.20 \%$ & -0.3 & 24.8 & 0.8 & 0.0 & 1.3 & 0.8 & 5.0 & 75.9 \\
\cline { 2 - 12 } & STR & 3.48 & $38.80 \%$ & -6.2 & 24.0 & -1.1 & 0.0 & 1.8 & 1.2 & 1.1 & 29.5 \\
\cline { 2 - 12 } & DV & 3.94 & $55.67 \%$ & -3.9 & 26.2 & 0.3 & -0.2 & 2.8 & 1.6 & 3.6 & 24.7 \\
\hline \hline \multirow{2}{*}{$心$} & MA & 3.38 & $2.53 \%$ & -32.6 & 4.5 & 0.9 & 0.0 & 1.9 & 1.1 & -7.0 & 119.0 \\
\cline { 2 - 11 } & STR & 3.88 & $0.80 \%$ & -28.0 & 5.9 & 1.6 & 0.0 & 2.2 & 1.7 & -3.0 & 45.1 \\
\cline { 2 - 11 } & DV & 2.69 & $60.53 \%$ & -6.4 & 35.8 & 0.3 & -0.2 & 2.3 & 1.2 & 5.5 & 59.1 \\
\hline
\end{tabular}

Table 5: Summary statistics for all hedges in both the UOC and DOP cases if the hedge portfolio is unwound at the observed spot $S_{\tau}$ after the barrier is breached. The mean absolute deviation about the median is labelled 'mad.'. All numbers except skewness, kurtosis and the loss probability are in percent of spot at initiation. The cost is computed from market prices.

\begin{tabular}{|c|c|c|c|c|c|c|c|c|c|c|c|}
\hline \multirow{3}{*}{$\begin{array}{l}0 \\
\wp \\
\wp \\
\rho\end{array}$} & $T^{i}$ & 1.0 & 1.0 & 1.0 & 1.0 & 1.0 & 0.75 & 0.75 & 0.75 & 0.5 & 0.5 \\
\hline & $K^{i}$ & 90.0 & 118.2 & 121.8 & 125.4 & 130.8 & 120.0 & 123.6 & 130.8 & 120.0 & 127.2 \\
\hline & $\overline{\alpha^{i}}$ & 1.0 & -17.70 & 13.62 & 12.91 & -13.35 & 1.74 & -2.14 & 3.64 & -0.06 & -0.11 \\
\hline \multirow{3}{*}{ ○े } & $\overline{T^{i}}$ & $\overline{1.0}$ & 1.0 & 1.0 & 1.0 & 1.0 & $\overline{0.75}$ & $\overline{0.75}$ & 0.75 & 0.5 & $\overline{0.5}$ \\
\hline & $K^{i}$ & 110.0 & 81.2 & 78.8 & 76.4 & 72.8 & 80.0 & 77.6 & 72.8 & 80.0 & 75.2 \\
\hline & $\alpha^{i}$ & 1.0 & -71.62 & 45.72 & 100.0 & -78.86 & 0.91 & -5.67 & 7.38 & 0.46 & 0.0 \\
\hline
\end{tabular}

Table 6: Specification of the sub-replication portfolio. Absolute portfolio weights $\alpha^{i}$ are computed according to robustification step $\mathrm{C}$ in Table 1. Hedge options are calls for UOC and puts for DOP. Strikes are in percent of the level of the underlying.

\begin{tabular}{|l||c|r||r|r|r|r|r|r|r|r|}
\hline & Mean cost & $P($ Gain $)$ & $\min$ & $\max$ & mean & med. & std. & mad. & skew. & kurt. \\
\hline UOC & 3.50 & $0.00 \%$ & -12.5 & 0.0 & -1.2 & 0.0 & 1.9 & 1.2 & -1.8 & 6.0 \\
DOP & 1.38 & $0.00 \%$ & -157.0 & 0.0 & -3.0 & 0.0 & 8.4 & 3.0 & -10.1 & 152.8 \\
\hline
\end{tabular}

Table 7: Summary statistics for sub-replicating MA hedges in both the UOC and DOP cases if the hedge portfolio is unwound at the observed spot $S_{\tau}$ after the barrier breach. The mean absolute deviation about the median is labelled 'mad.'. All numbers except skewness, kurtosis and the gain probability are in percent of spot at initiation. The cost is computed from market prices. 


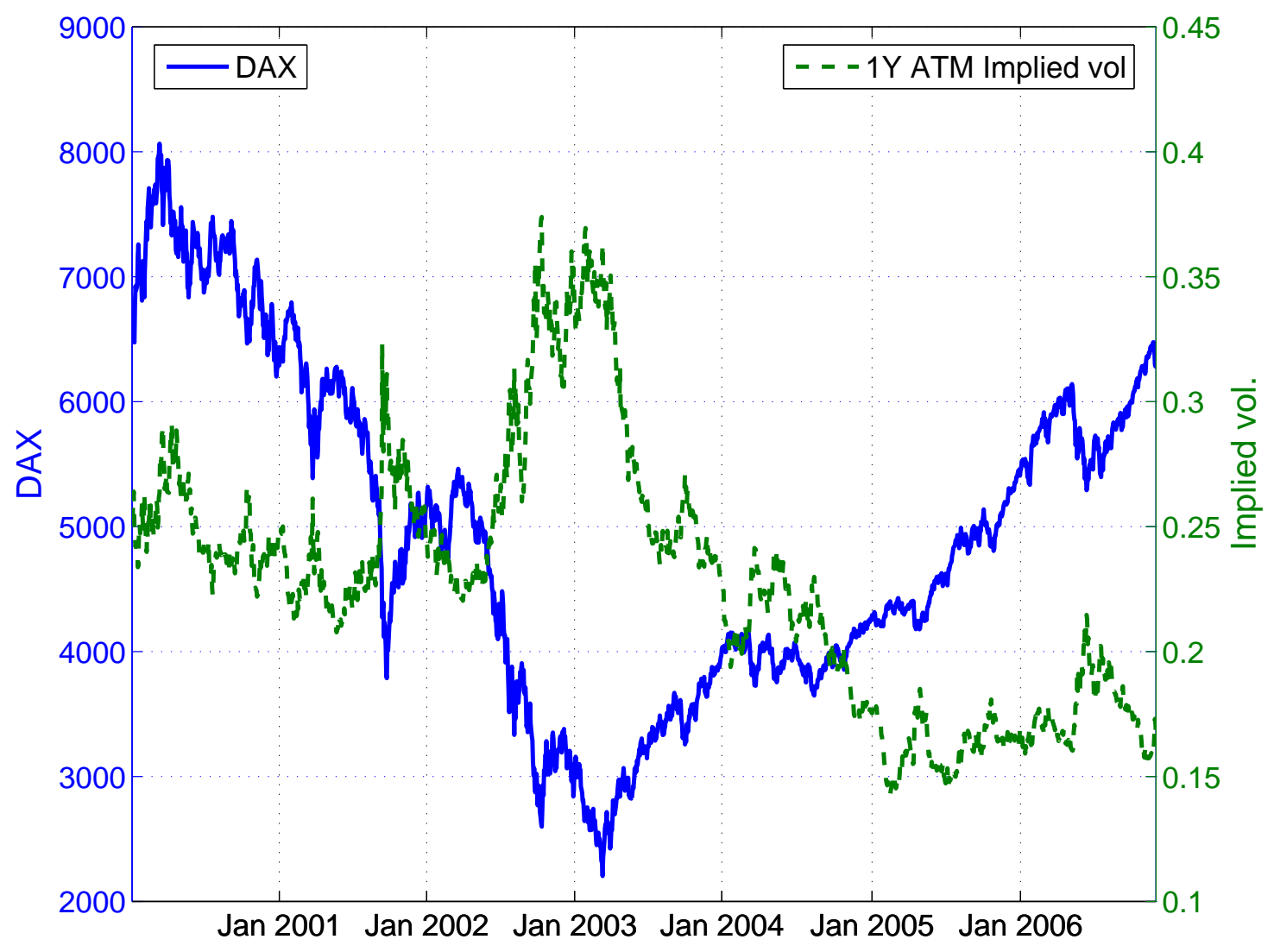

Figure 1: DAX index levels and the DAX 1-year at-the-money implied volatility. 


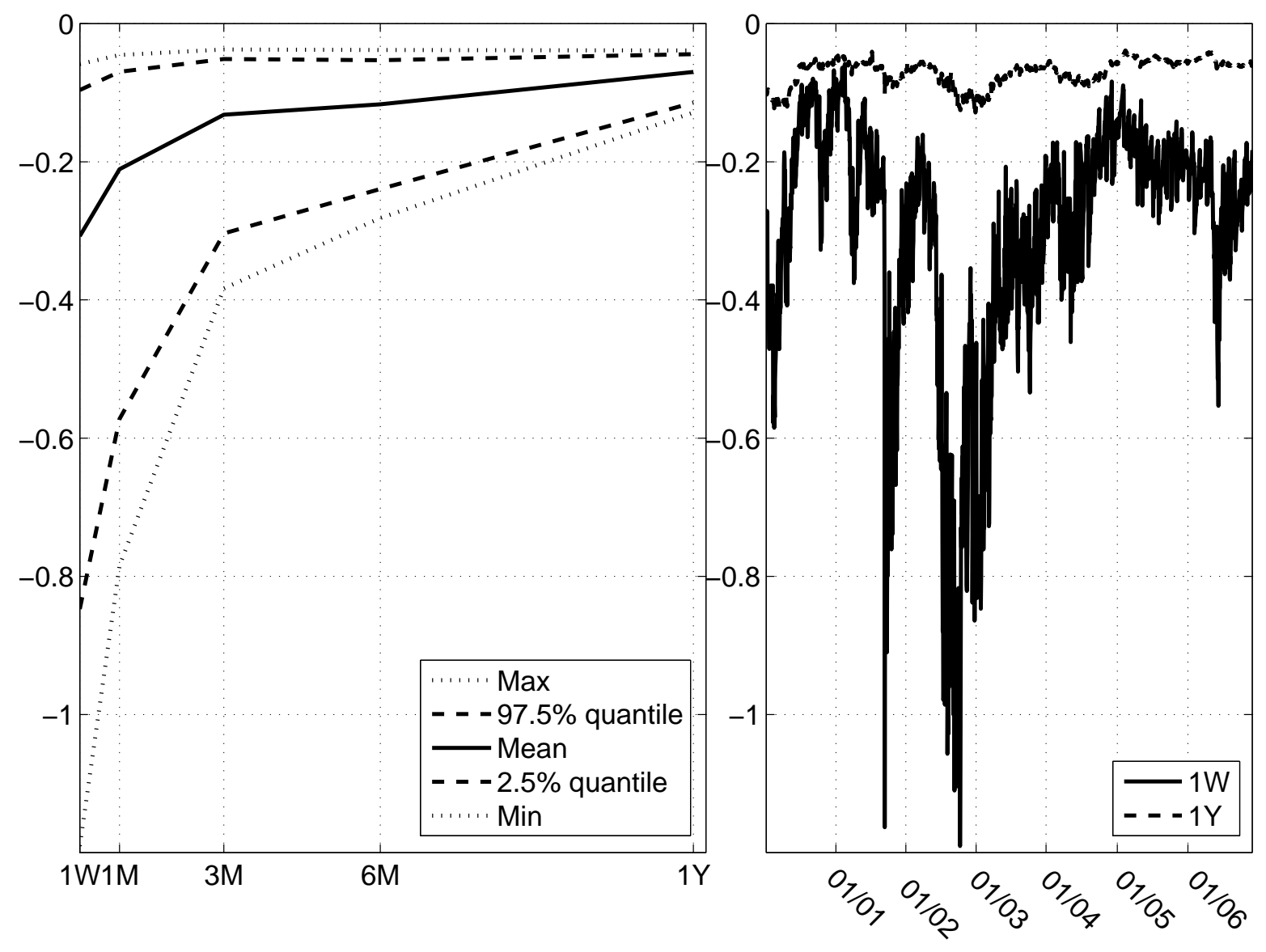

Figure 2: Average term structures and time series of the DAX at-the-money implied volatility skew $\left[\sigma^{2}\right.$ (Forward-ATM-level $\left.+0.01 \%\right)-\sigma^{2}($ Forward-ATM-level $\left.-0.01 \%)\right] / 0.02 \%$. 


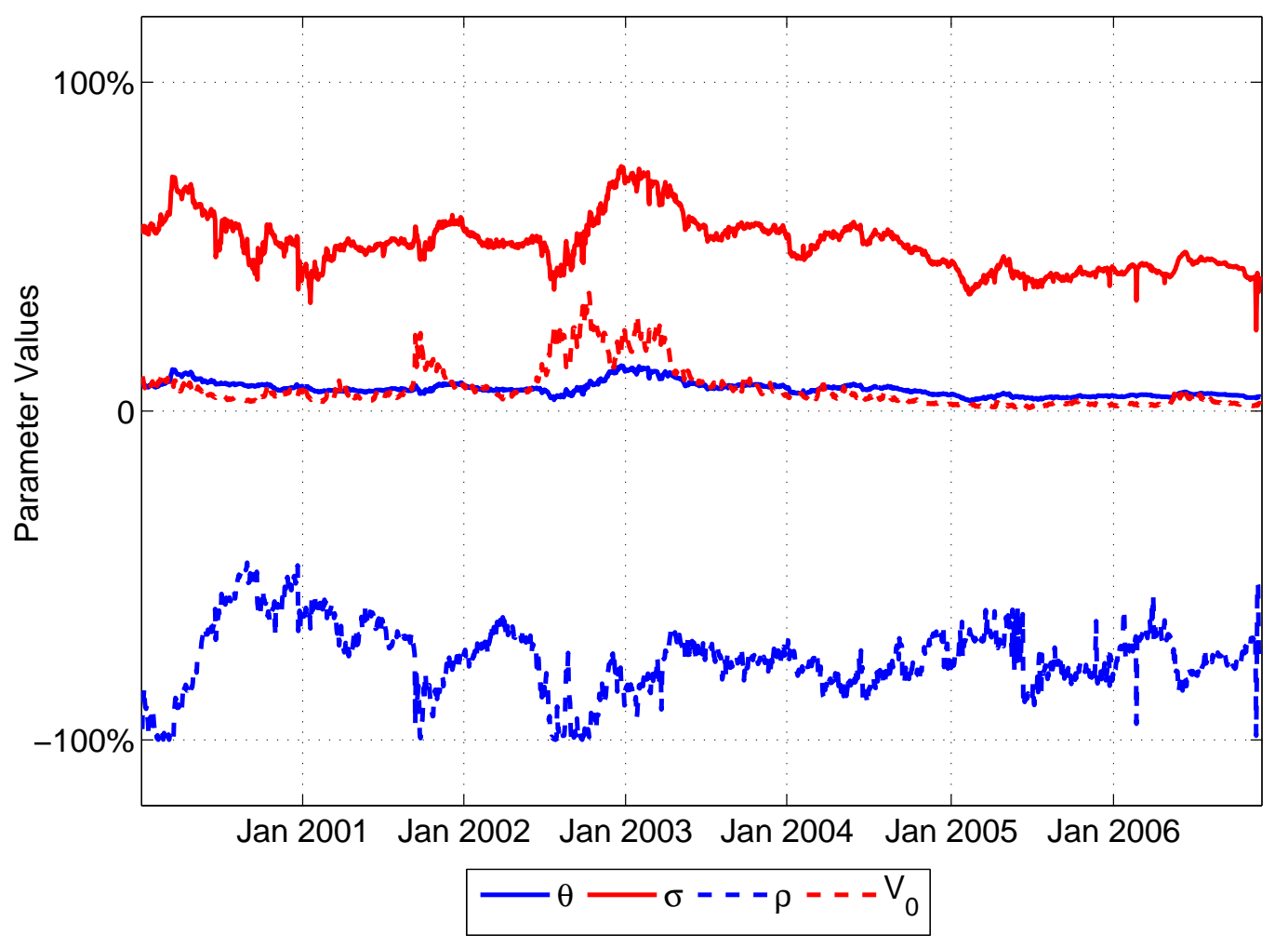

Figure 3: Time series of parameters in the Heston model when calibrated to daily DAX volatility surfaces. Speed of mean-reversion is fixed at $\kappa=2$ in the calibration, Feller condition is enforced. 

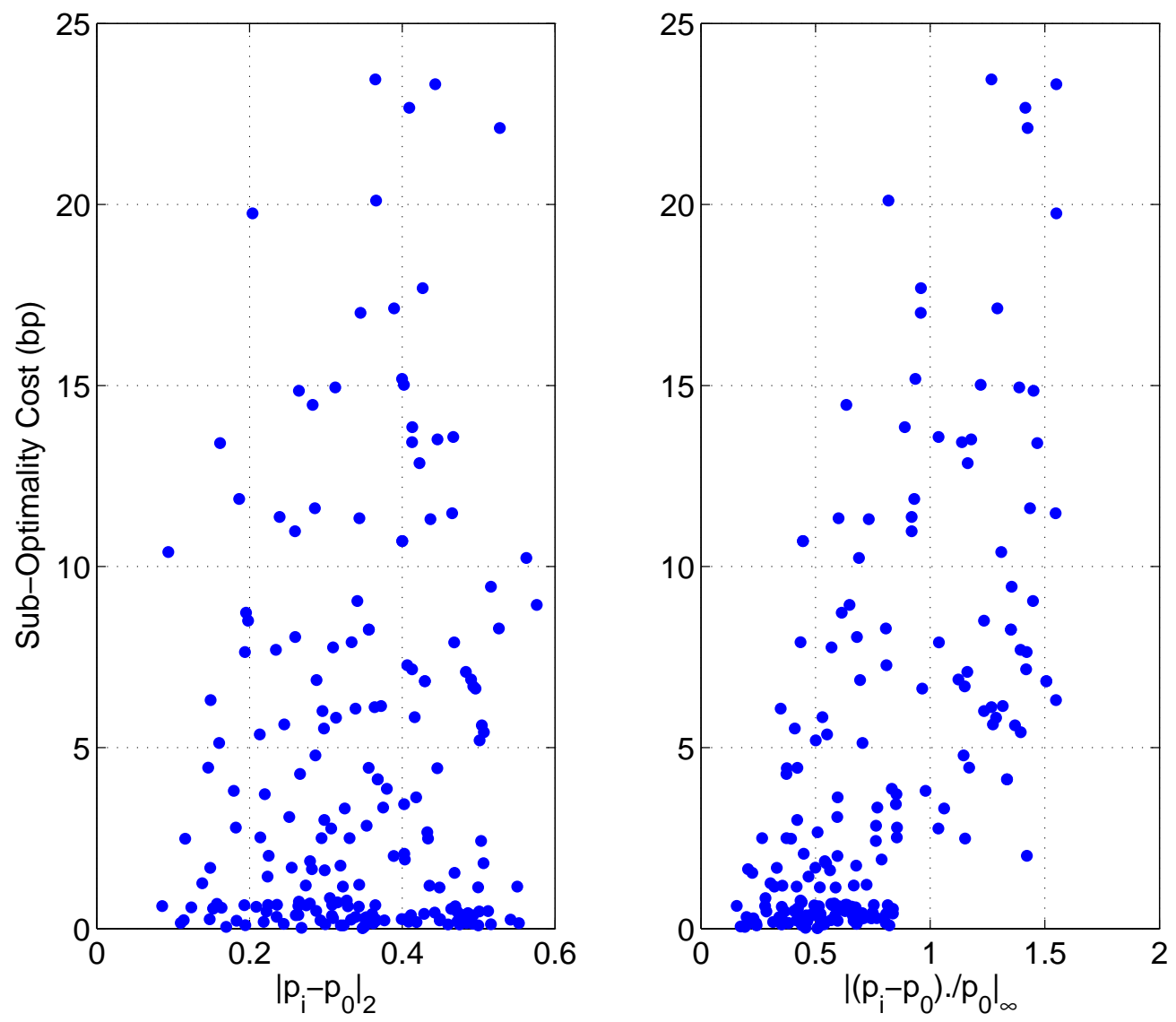

Figure 4: Sub-optimality costs in basis points for a fixed minimal assumptions hedge for the UOC under alternative market conditions. Plotted is Cost $_{0}-$ Cost $_{i}$ against the Euclidian norm of $p_{i}-p_{0}$ and the maximum norm of (elementwise) relative parameter differences. Cost are the costs for a static hedge based on a Heston parameter vector $p_{0}$, but valued at a randomly drawn parameter vector $p_{i}$. Cost ${ }_{i}$ are the costs for a static hedge based and priced on the Heston parameter vector $p_{i}$. The random vectors $p_{i}$ are drawn uniformly from the parameter hypercube $P$ in Table 1, The riskfree rate is set to $r(t)=3 \%$. 

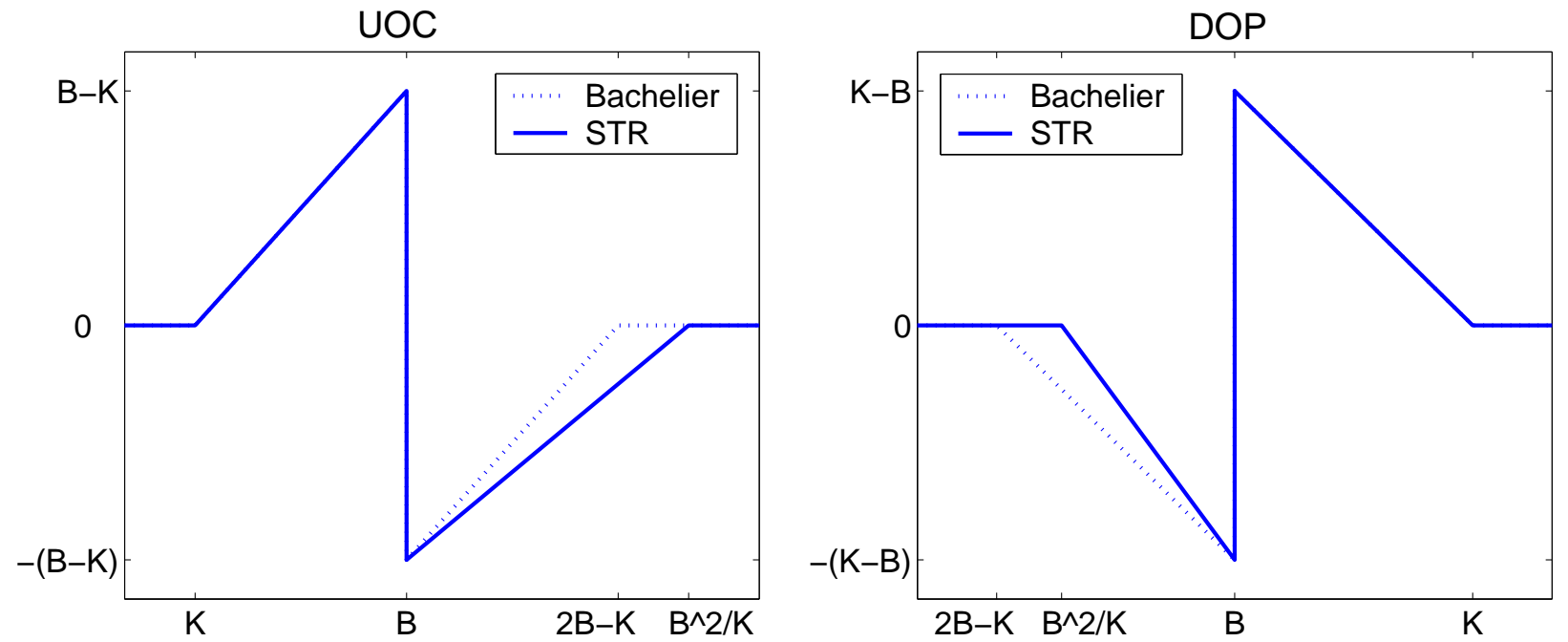

Figure 5: Adjusted payoff functions for UOC and DOP contracts of the STR hedge derived in the BS model with $r-q=0$ and the analogous replicating static hedge in the Bachelier model with zero drift.
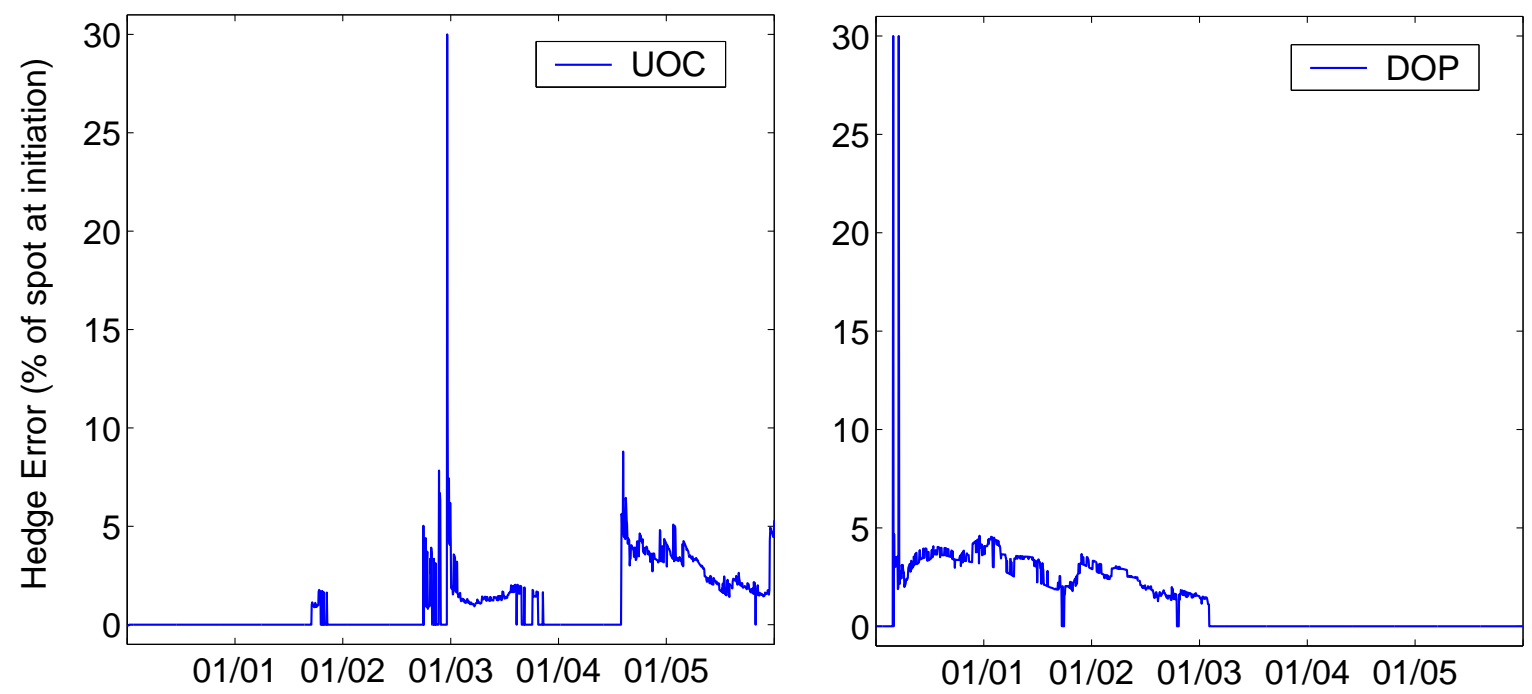

Figure 6: Time series of MA hedge errors for the UOC and DOP for the case of unwinding the hedge portfolio at the barrier $B$. 

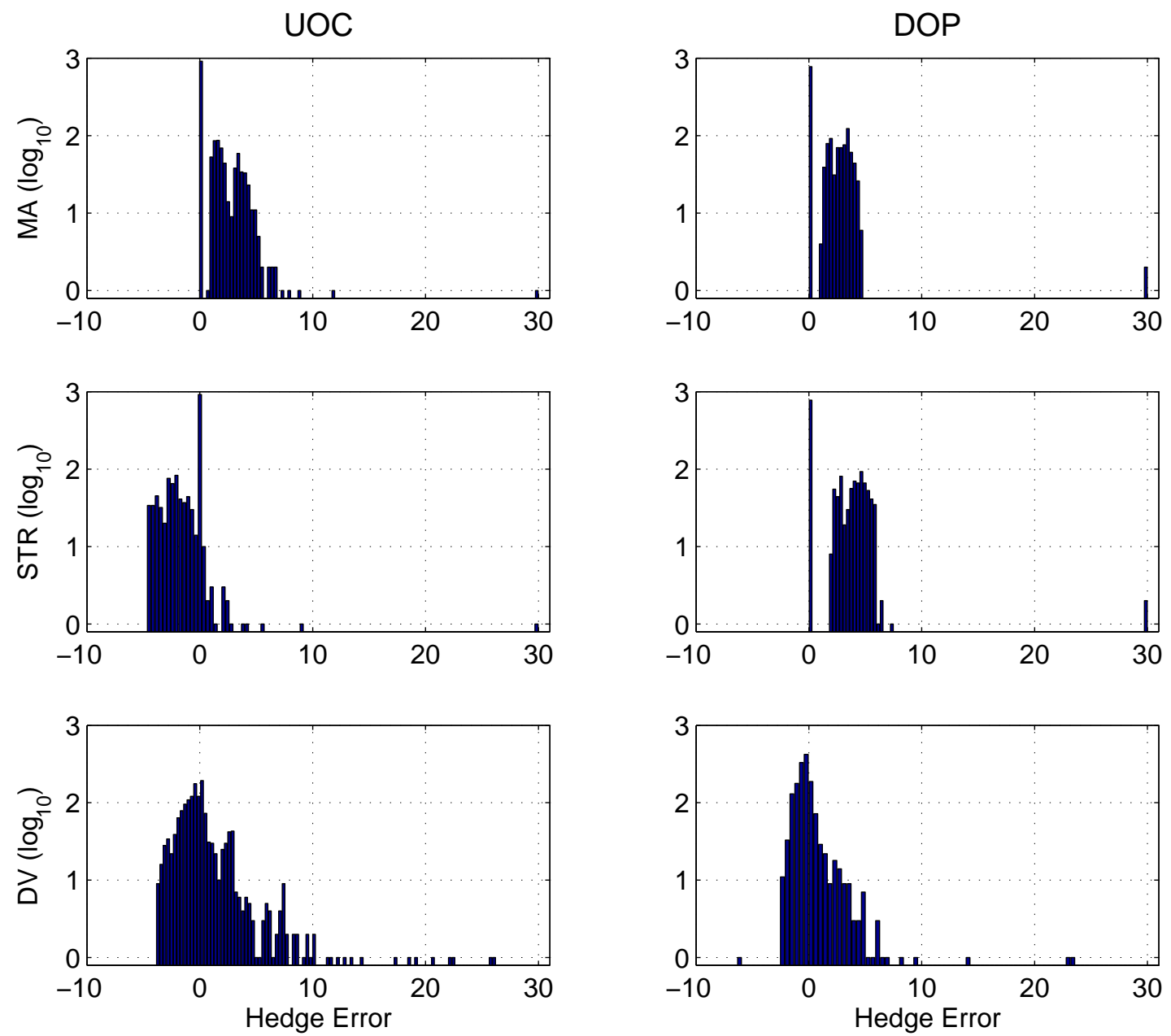

Figure 7: Hedge error frequencies for the MA, STR and DV hedges of UOC and DOP contracts if the hedge portfolio is unwound at the barrier $B$. Hedge errors are in percent of spot at initiation. 

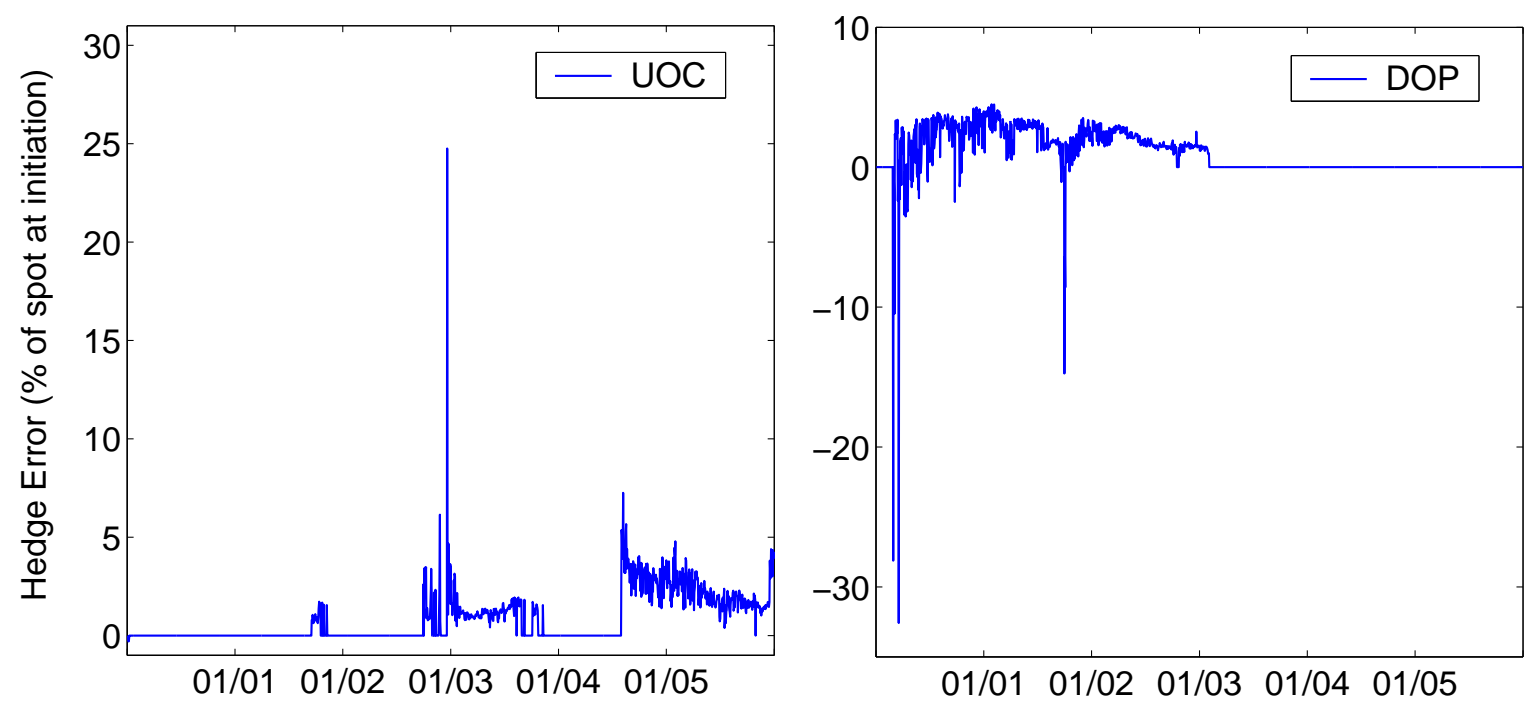

Figure 8: Time series of hedge errors for the UOC and DOP for the case of unwinding the hedge portfolio at the observed spot $S_{\tau}$, where $\tau$ is the stopping time at barrier hit. 